Baroclinic multipole formation from heton interaction

This article has been downloaded from IOPscience. Please scroll down to see the full text article.

2010 Fluid Dyn. Res. 42045501

(http://iopscience.iop.org/1873-7005/42/4/045501)

View the table of contents for this issue, or go to the journal homepage for more

Download details:

IP Address: 134.246.133.132

The article was downloaded on 28/04/2010 at 07:38

Please note that terms and conditions apply. 


\title{
Baroclinic multipole formation from heton interaction
}

\author{
Mikhail A Sokolovskiy $^{1}$ and Xavier J Carton ${ }^{2}$ \\ ${ }^{1}$ Water Problems Institute of RAS, 3, Gubkina Street, 119333, Moscow, Russia \\ ${ }^{2}$ Laboratoire de Physique des Océans, UFR Sciences, UBO, 6 Av. Le Gorgeu, \\ 29200 Brest, France \\ E-mail: sokol@aqua.laser.ru
}

Received 23 April 2009

Published 14 April 2010

Online at stacks.iop.org/FDR/42/045501

Communicated by Y Hayashi

\begin{abstract}
In a two-layer quasi-geostrophic model, the interaction between two oppositesigned hetons (baroclinic vortex pairs) is studied analytically and numerically, for singular and finite-area vortices.

For point vortices, using trilinear coordinates, it is shown that the possible evolutions depend on the deformation radius $R_{\mathrm{d}}$ : for large $R_{\mathrm{d}}$, the layers decouple, vortices pair in each layer and their trajectories are open; for medium $R_{\mathrm{d}}$, the exchange of opposite-sign partners between layers becomes possible; for small $R_{\mathrm{d}}$, two other regimes appear: one where hetons remain unaltered during their evolution but follow open trajectories, and one where hetons occupy only a bounded subdomain of space at all times. Conditions for invariant co-rotation of the heton pair are derived and analyzed.

Then, the nonlinear evolutions of finite-area heton pairs, with piecewiseconstant vorticity, are computed with contour dynamics. When the central cyclonic vortex is initially aligned vertically, a transition occurs between three nonlinear regimes as layer coupling increases: for weak coupling, the vortices pair horizontally and drift away in opposite directions; for moderate layer coupling, the core vortex splits into two parts, one of which remains as a tilted columnar vortex at the center; for stronger layer coupling, each anticyclone pairs with part of the cyclone in each layer, thus forming an L-shaped dipole, a new coherent structure of two-layer flows. When the initial distance between the central and satellite vortices is increased, the velocity shear at the center decreases and the central vortex remains vertically aligned, thus forming a Z-shaped tripole, also a newly observed vortex compound. Such tripoles also compete with oscillating states, in which the core vortex periodically aligns and tilts, a regime observed when layer coupling is moderate and as vortices become closer in each layer. This Z-shaped tripole forms for various values of stratification and of initial distances between vortices, and is therefore a robust vortex compound in two-layer quasi-geostrophic flows.
\end{abstract}




\section{Introduction}

Vortices are coherent structures that play a central role in many fluid flows, in nature or in the laboratory. Two well-known examples of this role are two-dimensional turbulence and planetary flows. In two-dimensional incompressible turbulence and in strongly rotating stratified turbulence, vortex interactions have been associated with upscale energy transfer, and the filaments resulting from these interactions materialize the downscale enstrophy cascade (McWilliams 1984, 1989, 1990a, 1990b). In the atmosphere and oceans, models and observations have established how efficient vortices are in carrying momentum, heat or tracers across the dynamical barriers of intense jets (see Carton (2001) for a review). Vortices also play a central role in the mixing of such tracers. This efficiency of vortices to carry or mix fluids is related to their internal structure: vortices are intense, horizontal recirculation motions that trap fluid in their core and mix them at their periphery; their internal motion is much stronger than external advecting velocities; their lifetime is much longer than their internal turnover period. The dual aspect of intense vorticity and of fluid trapping in the vortex core is described by a single variable in stratified, rotating fluid models: potential vorticity. Vortices are associated with a significant potential vorticity anomaly between their core and their surrounding.

Vortices come in various shapes, associated with a monopolar or a multipolar distribution of potential vorticity anomaly. The monopolar vortex has been historically studied for its simplicity and ubiquity. Much theoretical, numerical and experimental work of the past 20 years has shown that monopolar vortices can be unstable in both homogeneous and rotating, stratified fluids; this instability can form dipoles, tripoles and quadrupoles, in decreasing order of frequency (e.g. Gryanik 1983a, 1983b, Kozlov and Makarov 1985, Pedlosky 1985, Kozlov et al 1986, Flierl 1988, Helfrich and Send 1988, Carton and McWilliams 1989, 1996, Polvani and Carton 1990, Orlandi and van Heijst 1992, Kozlov 1994, Morel and Carton 1994, Sokolovskiy 1997a, 1997b, Carton and Corréard 1998, Corréard and Carton 1999, Baey and Carton 2002, Jamaloodeen and Newton 2007, Reznik and Kizner 2007).

In a multilayer fluid, a specific dipole, consisting of a cyclone and an anticyclone lying in different layers, is particularly important due to its ability to carry heat. This dipolar structure, called a heton, was first introduced by Hogg and Stommel in 1985 to describe a point vortex pair associated with a thermal anomaly. Indeed, under the geostrophic and hydrostatic approximations, conventional for geophysical fluid dynamics (Pedlosky 1987), an anticyclone in an upper layer or a cyclone in a lower layer induces a downward displacement of the density interface between layers, and therefore a positive thermal anomaly. Conversely, a negative thermal anomaly is created by reversing the vortex polarities. Now, the association of two such vortices (with like-signed thermal anomalies) is able to self-advect when the vortices are not vertically aligned. This ability to transport heat was the key issue described by Hogg and Stommel (1985a) for point vortices. After this seminal paper, the notion of heton was generalized to vortices with finite horizontal dimensions (in particular, vortex patches with uniform potential vorticity).

Hetons are not purely theoretical constructions: they can form from unstable jets in the deep ocean (Meacham 1991, Flierl et al 1999). Indeed, they have been observed south of the Gulf Stream; they are composed of a Gulf Stream cold-core ring associated with a Sargasso Sea water anticyclone. Hetons can also form from the instability of coastal currents (Morel and McWilliams 2001, Chérubin et al 1997). Observations of Mediterranean water eddies south and west of the Iberian Peninsula show that deep anticyclones (meddies) can be associated with cyclones above or below them, thus forming warm or cold hetons (Chérubin et al 1997, Takahashi and Masuda 1998, Paillet et al 2002). Observations and 
models have also shown that hetons can originate in the baroclinic instability of convective columns (Legg and Marshall 1993). Hetons also represent vortex pairs under sea ice (Chao and Shaw 1999a, 1999b, 2000, 2003), vortices resulting from the interaction of oceanic currents with bottom topography (Sokolovskiy et al 2001) or tropical cyclones and hurricanes in the atmosphere (Pedlosky 1985, Pullin 1992, Flatau et al 1994, Tevs 1999). In summary, many observations of oceanic and atmospheric vortices confirm the existence of hetons. Hetons can also form from horizontal and vertical vortex interactions, such as merger and alignment, when the original vortices are composed of opposite-signed poles of potential vorticity (Verron et al 1990, Verron and Valcke 1994, Corréard and Carton 1998).

Previous studies have shown that heton interactions can scatter these dipoles or form new multipoles (Hogg and Stommel 1985b, Valcke and Verron 1993, Sokolovskiy and Verron 2000a, 2000b, 2002a, 2002b, 2004, 2006, Gryanik et al 2006, Kizner 2006). But none of these studies have considered the interaction of a cold and a warm heton. This configuration is not only particularly important for oceanic vortices near the Iberian Peninsula, but also, in view of vertical vortex alignment, an essential process in rotating stratified turbulence.

Indeed, a previous study (Corréard and Carton 1998) has investigated the influence of the initial distribution of potential vorticity anomalies on the alignment process. This study considered two vortices initially with uniform relative vorticity (the so-called RVI (relative vorticity intensity) conditions). Each vortex was lying in a separate layer. The corresponding potential vorticity distribution is that of two opposite-signed hetons, vertically asymmetric and with non-uniform potential vorticity distribution. This study showed that for strong layer coupling, initially close vortices align and form a baroclinic tripole (a central, vertically aligned, vortex column, with one opposite-signed satellite in each layer, the whole structure being Z-shaped) and initially distant vortices diverge as two hetons. On the contrary, for weaker layer coupling, initially close vortices oscillate around the center of the plane and more distant vortices end up as scattering horizontal dipoles.

Considering that (a) warm and cold hetons co-exist in the ocean and can interact, (b) the alignment of RVI vortices allows the interaction of warm and cold hetons, (c) the interaction of two opposite-signed (and symmetrical) hetons with zero linear momentum is likely to produce more complex baroclinic multipoles (in particular rotating structures) and (d) the knowledge and classification of baroclinic multipoles, contrary to their 2D counterparts, are far from complete, the present study addresses the interaction between two opposite-signed hetons with focus on nonlinear evolution and baroclinic multipole formation. This evolution is studied here by analytical and numerical methods when the hetons are point-like (section 3), and by a contour-dynamics approach for finite-core hetons (section 4). Conclusions are drawn on how the results of this study shed new light on previous observations.

\section{Mathematical and physical framework}

When flows of stratified, rotating, incompressible fluid have small Rossby number and order unity Burger number, the quasi-geostrophic approximation is valid. In the absence of forcing and dissipation, two-layer quasi-geostrophic flows are governed by the potential vorticity equation:

$$
\partial_{t} q_{j}+J\left(\psi_{j}, q_{j}\right)=0
$$

where

$$
q_{j}=\nabla^{2} \psi_{j}+F_{j}\left(\psi_{k}-\psi_{j}\right)+f_{0}
$$


is potential vorticity in layer $j=1,2$ (top, bottom) and $k=3-j$. The first term is relative vorticity of the vortices, the second term is vorticity associated with vertical stretching of water columns, and the third term is ambient vorticity (due to global rotation), also called Coriolis parameter. The symbols $\nabla^{2}$ and $J$ are the 2D operators of Laplace and Jacobi, respectively; the layerwise streamfunction is $\psi_{j}$, and $F_{j}=f_{0}^{2} / g^{\prime} h_{j}$ are the layer coupling coefficients. The Coriolis parameter $f_{0}$ is constant. The reduced gravity $g^{\prime}=g \Delta \rho / \rho$ is much smaller than the usual gravity because of the weak relative density difference between layers: $\Delta \rho=\rho_{2}-\rho_{1} \ll \rho_{1}$. The thickness of layer $j$ at rest is $h_{j}$ and $h=h_{1}+h_{2}$ is the total fluid thickness. The internal radius of deformation is defined by

$$
R_{\mathrm{d}}=\sqrt{g^{\prime} h_{1} h_{2}} /\left(f_{0} \sqrt{h}\right) .
$$

For convenience, we write $\gamma=1 / R_{\mathrm{d}}$. Finally, the horizontal velocity components are

$$
u_{j}=-\frac{\partial \psi_{j}}{\partial y}, \quad v_{j}=\frac{\partial \psi_{j}}{\partial x} .
$$

In the present study, two idealizations of potential vorticity distributions will be used:

- point-like vortices with constant intensity $\kappa_{j}^{\alpha}$,

$$
q_{j}(x, y)=\sum_{\alpha} \kappa_{j}^{\alpha} \delta\left(x-x_{j}^{\alpha}\right) \delta\left(y-y_{j}^{\alpha}\right),
$$

so that, strictly speaking, each point vortex of the $j$ th layer represents a vertical vortex segment between the quasi-horizontal boundaries of this layer (see figure 1), and

- finite-area, piecewise-constant vortices with constant potential vorticity $q_{j}^{\alpha}$ in the finite domains $S_{j}^{\alpha}$ bounded by contours $C_{j}^{\alpha}$,

$$
q_{j}(x, y)=\sum_{\alpha} q_{j}^{\alpha} \Theta\left(S_{j}^{\alpha}\right)
$$

where $\Theta\left(S_{j}^{\alpha}\right)$ is the step function, equal to 1 inside and 0 outside the domain $S_{j}^{\alpha}$.

In each case, two vortices with equal and opposite polarity lie in each layer. Here, the lower index refers to layer number $(j=1,2)$ and the upper index to vortex number in the layer.

Under these assumptions, the streamfunction corresponding to finite-core vortices is

$\psi_{j}(x, y)=\sum_{\alpha=1}^{2} h_{j} q_{j}^{\alpha} \oint_{C_{j}^{\alpha}} w\left[W(r)+\frac{h_{3-j}}{h_{j}} W_{0}(r)\right] \mathrm{d} \nu+\sum_{\alpha=1}^{2} h_{3-j} q_{3-j}^{\alpha} \oint_{C_{3-j}^{\alpha}} w\left[W(r)-W_{0}(r)\right] \mathrm{d} v$.

In (3), the notations

$$
\begin{aligned}
& w=\frac{\left(x^{\prime}-x\right) \partial y^{\prime} / \partial v-\left(y^{\prime}-y\right) \partial x^{\prime} / \partial v}{r^{2}} \\
& W=\frac{r^{2}}{4 \pi}\left(\ln r-\frac{1}{2}\right), \quad W_{0}=\frac{1}{2 \pi \gamma^{2}}\left[\gamma r K_{1}(\gamma r)-1\right]
\end{aligned}
$$

are introduced, and $v$ is a linear parameter measured counterclockwise along each contour $C_{j}^{\alpha} ; K_{1}$ here, and $I_{1}$ in (6), are the modified Bessel functions of the second kind and first kind, respectively.

The velocity of a fluid particle with label $n$ on contour $C_{j}^{\alpha}$ of any finite-area piecewiseconstant vortex is obtained by solving the equations

$$
\frac{\mathrm{d}}{\mathrm{d} t}\left(x_{j}^{\alpha}\right)_{n}=\left(u_{j}^{\alpha}\right)_{n}, \quad \frac{\mathrm{d}}{\mathrm{d} t}\left(y_{j}^{\alpha}\right)_{n}=\left(v_{j}^{\alpha}\right)_{n},
$$


with the initial conditions

$$
t=0: \quad\left(x_{j}^{\alpha}\right)_{n}=\left(x_{j}^{\alpha}\right)_{n}^{0}, \quad\left(y_{j}^{\alpha}\right)_{n}=\left(y_{j}^{\alpha}\right)_{n}^{0} .
$$

Here, $j=1,2 ; \alpha=1,2$, and $n=1, \ldots, N_{j}^{\alpha}$. The expressions for velocities on the right-hand sides of (4) are obtained after substitution of (3) into (2).

Equations (4) and (5) are the basis for a two-layer modification of the contour dynamics method, including contour surgery (see Kozlov et al 1986, Makarov 1991, Sokolovskiy 1997a, 1997b; Sokolovskiy and Verron 2000a). The system of ordinary differential equations (4) and (5) is solved with a fourth-order Runge-Kutta method using the Gill 'optimum' formula for timestepping (Hairer et al 1987). An initial number of nodes $N_{j}^{\alpha}=120$ is typically used for a circle of unit radius $(j=1,2$ and $\alpha=1,2)$. During the calculation, this number increases proportionally to the length of the contour; new nodes are uniformly redistributed along the contour. The approximation, interpolation, numerical differentiation and grid integration procedures are carried out using periodic cubic spline techniques (Kozlov 1983). Makarov's algorithm (1991) of contour surgery allows vorticity filament separation and contour reconnection when vortices with the same potential vorticity values approach each other. Thus, this method for the calculation of finite-time, finiteamplitude evolution of vortices leads to minimal numerical dissipation, and has been shown to compare well to spectral or gridded methods (Corréard and Carton 1999).

In numerical calculations, the non-dimensional time unit is half a rotation period on the unit radius contour for a heton with vertical axis. This requirement implies a condition on the choice of constants $q_{j}^{\alpha}$ :

$$
\left|q_{j}^{\alpha}\right|=\frac{\left(1+h_{3-j} / h_{j}\right) I_{1}(\gamma) K_{1}(\gamma)}{2 \pi} .
$$

In the point vortex limit

$$
\lim _{\substack{S_{j}^{\alpha} \rightarrow 0 \\ q_{j}^{\alpha} \rightarrow \infty}} q_{j}^{\alpha} S_{j}^{\alpha}=\kappa_{j}^{\alpha},
$$

point vortex motion obeys equations derived from (2) and (3):

$$
\begin{aligned}
\dot{x}_{j}^{\alpha}=-\frac{h_{j}}{2 \pi}\left\{\sum_{\substack{\beta=1 \\
\beta \neq \alpha}}^{2} \kappa_{j}^{\beta} \frac{y_{j}^{\alpha}-y_{j}^{\beta}}{\left(r_{j j}^{\alpha \beta}\right)^{2}}\left[1+\frac{h_{3-j}}{h_{j}} \gamma r_{j j}^{\alpha \beta} K_{1}\left(\gamma r_{j j}^{\alpha \beta}\right)\right]\right. \\
\left.+\sum_{\beta=1}^{2} \kappa_{3-j}^{\beta} \frac{h_{3-j}}{h_{j}} \frac{y_{j}^{\alpha}-y_{3-j}^{\beta}}{\left(r_{j(3-j)}^{\alpha \beta}\right)^{2}}\left[1-\gamma r_{j(3-j)}^{\alpha \beta} K_{1}\left(\gamma r_{j(3-j)}^{\alpha \beta}\right)\right]\right\}, \\
\dot{y}_{j}^{\alpha}=\frac{h_{j}}{2 \pi}\left\{\sum_{\substack{\beta=1 \\
\beta \neq \alpha}}^{2} \kappa_{j}^{\beta} \frac{x_{j}^{\alpha}-x_{j}^{\beta}}{\left(r_{j j}^{\alpha \beta}\right)^{2}}\left[1+\frac{h_{3-j}}{h_{j}} \gamma r_{j j}^{\alpha \beta} K_{1}\left(\gamma r_{j j}^{\alpha \beta}\right)\right]\right. \\
\left.+\sum_{\beta=1}^{2} \kappa_{3-j}^{\beta} \frac{h_{3-j}}{h_{j}} \frac{x_{j}^{\alpha}-x_{3-j}^{\beta}}{\left(r_{j(3-j)}^{\alpha \beta}\right)^{2}}\left[1-\gamma r_{j(3-j)}^{\alpha \beta} K_{1}\left(\gamma r_{j(3-j)}^{\alpha \beta}\right)\right]\right\} .
\end{aligned}
$$

Here, $r_{i j}^{\alpha \beta}=\sqrt{\left(x_{i}^{\alpha}-x_{j}^{\beta}\right)^{2}+\left(y_{i}^{\alpha}-y_{j}^{\beta}\right)^{2}}$. The trajectories of point vortices are integrated with the fourth-order Runge-Kutta method. 


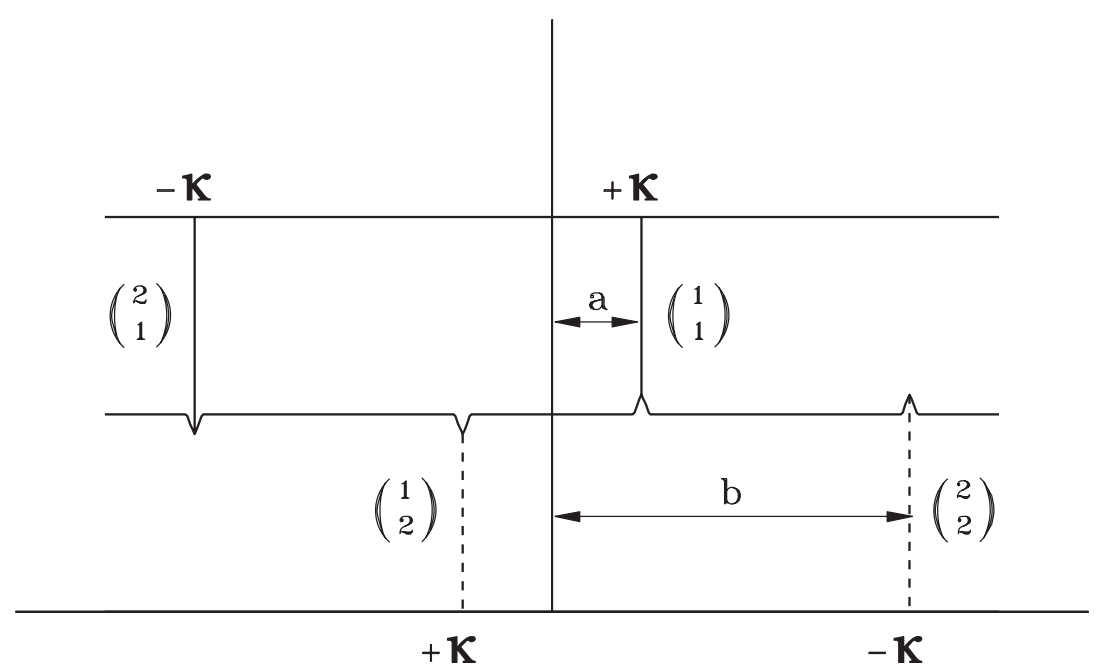

Figure 1. Vertical section of the general colinear configuration under study. Initial positions and strengths of vortices (vortex lines) are indicated; the lower index refers to the vortex layer, and the upper index to its number in the layer. Thick solid lines correspond to the vortex lines in the upper layer, and dashed ones to the vortex lines in the lower layer. Each vortex generates a local deformation of the density interface related to its sign. Note that the vortices can also have a finite extent (with radius $R$ in section 4 of the study). The thin vertical line is the center of the plane.

When choosing a time scale for point vortices, we use the 'equivalence' principle of Polvani (1991). This principle states that the azimuthal velocity along a unit circle, induced by point vortex with strength $\kappa_{j}^{\alpha}$, is equal to the velocity along the contour of the finite-core vortex with potential vorticity (6) and of the same radius:

$$
\kappa_{j}^{\alpha}=\frac{2 \pi I_{1}(\gamma)}{\gamma q_{j}^{\alpha}} .
$$

\section{Point vortex modeling}

We study the interactions between two opposite-signed point vortex hetons in a two-layer fluid. Figure 1 shows the initial vortex configuration in the general case (with vertical tilt). We recall that, according to the Hogg and Stommel (1985a) classification, the vortex configurations that we may obtain correspond to hetons $\left(\begin{array}{l}2 \\ 1\end{array}\right)\left(\begin{array}{l}1 \\ 2\end{array}\right)$ and $\left(\begin{array}{l}1 \\ 1\end{array}\right)\left(\begin{array}{l}2 \\ 2\end{array}\right)$, antihetons $\left(\begin{array}{l}1 \\ 1\end{array}\right)\left(\begin{array}{l}1 \\ 2\end{array}\right)$ and $\left(\begin{array}{l}2 \\ 1\end{array}\right)\left(\begin{array}{l}2 \\ 2\end{array}\right)$, and horizontal dipoles $\left(\begin{array}{l}2 \\ 1\end{array}\right)\left(\begin{array}{l}1 \\ 1\end{array}\right)$ and $\left(\begin{array}{l}1 \\ 2\end{array}\right)\left(\begin{array}{l}2 \\ 2\end{array}\right)$. If the polarity of the vortices is that shown in figure 1 , the heton $\left(\begin{array}{l}2 \\ 1\end{array}\right)\left(\begin{array}{l}1 \\ 2\end{array}\right)$ is hot and the heton $\left(\begin{array}{l}1 \\ 1\end{array}\right)\left(\begin{array}{l}2 \\ 2\end{array}\right)$ is cold. If $a=b$, we have two hetons with 'vertical axes'; if $a \neq b$, the hetons have 'tilted axes'. More complex vortex compounds were not classified by Hogg and Stommel. The vortex strengths are

$$
\kappa_{1}^{1}=\kappa_{2}^{1}=-\kappa_{1}^{2}=-\kappa_{2}^{2}=\kappa>0 .
$$

All vortex positions are calculated with respect to the center of the plane. The symmetry of the problem (with $h_{1}=h_{2}=1 / 2$ ) ensures that at all times

$$
x_{1}^{1}=-x_{2}^{1}, \quad y_{1}^{1}=-y_{2}^{1}, \quad x_{1}^{2}=-x_{2}^{2}, \quad y_{1}^{2}=-y_{2}^{2} .
$$

At $t=0$, one has

$$
x_{1}^{1}=a, \quad x_{2}^{2}=b, \quad y_{1}^{1}=y_{1}^{2}=0 .
$$


To determine the dominant mechanism in the interaction between vortices (intra-layer or interlayer ones), parameters $a, b$ and $\gamma$ obviously play a key role.

\subsection{Hamiltonian, integral invariants and particular solutions}

The Hamiltonian of the problem is

$$
\begin{aligned}
H=-\frac{1}{2 \pi} \sum_{j=1}^{2} & h_{j}\left\{\sum_{\substack{\alpha, \beta=1 \\
\beta \neq \alpha}}^{2} \kappa_{j}^{\alpha} \kappa_{j}^{\beta}\left[\ln r_{j j}^{\alpha \beta}-\frac{h_{3-j}}{h_{j}} K_{0}\left(\gamma r_{j j}^{\alpha \beta}\right)\right]\right. \\
& \left.+\sum_{\alpha, \beta=1}^{2} \frac{h_{3-j}}{h_{j}} \kappa_{j}^{\alpha} \kappa_{3-j}^{\beta}\left[\ln r_{j(3-j)}^{\alpha \beta}+K_{0}\left(\gamma r_{j(3-j)}^{\alpha \beta}\right)\right]\right\} .
\end{aligned}
$$

The initial prescription of the vortex strengths and positions provides the value of the Hamiltonian. The equations of motion for each point vortex are written as

$$
\dot{q}_{j}^{\alpha}=\frac{\partial H}{\partial p_{j}^{\alpha}}=J\left(q_{j}^{\alpha}, H\right), \quad \dot{p}_{j}^{\alpha}=-\frac{\partial H}{\partial q_{j}^{\alpha}}=J\left(p_{j}^{\alpha}, H\right),
$$

with $q_{j}^{\alpha}=x_{j}^{\alpha}$ the generalized coordinates and $p_{j}^{\alpha}=x_{j}^{\alpha} \kappa_{j}^{\alpha} / 2$ the conjugate generalized momenta.

The other integral invariants are volume-integrated potential vorticity, the two components of linear momentum and angular momentum:

$$
\begin{aligned}
& Q=\sum_{j=1}^{2} h_{j} \sum_{\alpha=1}^{2} \kappa_{j}^{\alpha}, \quad P_{x}=\sum_{j=1}^{2} h_{j} \sum_{\alpha=1}^{2} \kappa_{j}^{\alpha} x_{j}^{\alpha}, \quad P_{y}=\sum_{j=1}^{2} h_{j} \sum_{\alpha=1}^{2} \kappa_{j}^{\alpha} y_{j}^{\alpha} \\
& M=\sum_{j=1}^{2} h_{j} \sum_{\alpha=1}^{2} \kappa_{j}^{\alpha}\left[\left(x_{j}^{\alpha}\right)^{2}+\left(y_{j}^{\alpha}\right)^{2}\right] .
\end{aligned}
$$

With our choice of conditions (9)-(11),

$$
Q=P_{x}=P_{y}=0 \quad \text { and } \quad M=\kappa\left(a^{2}-b^{2}\right) .
$$

Because of (13), a reduction to a three-vortex problem is possible, and the motion is integrable (Aref and Stremler 1999, Sokolovskiy and Verron 2000b). It can be analyzed with the use of trilinear coordinates (Aref and Stremler 1999).

But, first, we provide particular solutions:

- When $a=b$, we have two vertical hetons of opposite sign (or two antihetons if vertical symmetry is imposed). This interaction (exchange by partners between hetons) was studied by Hogg and Stommel (1985a).

- If $a=b$ and vertical symmetry of the vortex strength are imposed, quasi-barotropic vortex pairs move along the $y$-axis with velocity

$$
\dot{y}_{1}^{2}=\dot{y}_{2}^{2}=\dot{y}_{1}^{1}=\dot{y}_{2}^{2}=-\frac{\kappa \gamma}{2 \pi} K_{1}(2 \gamma a) \text {. }
$$


$\mathrm{B}, \Omega$

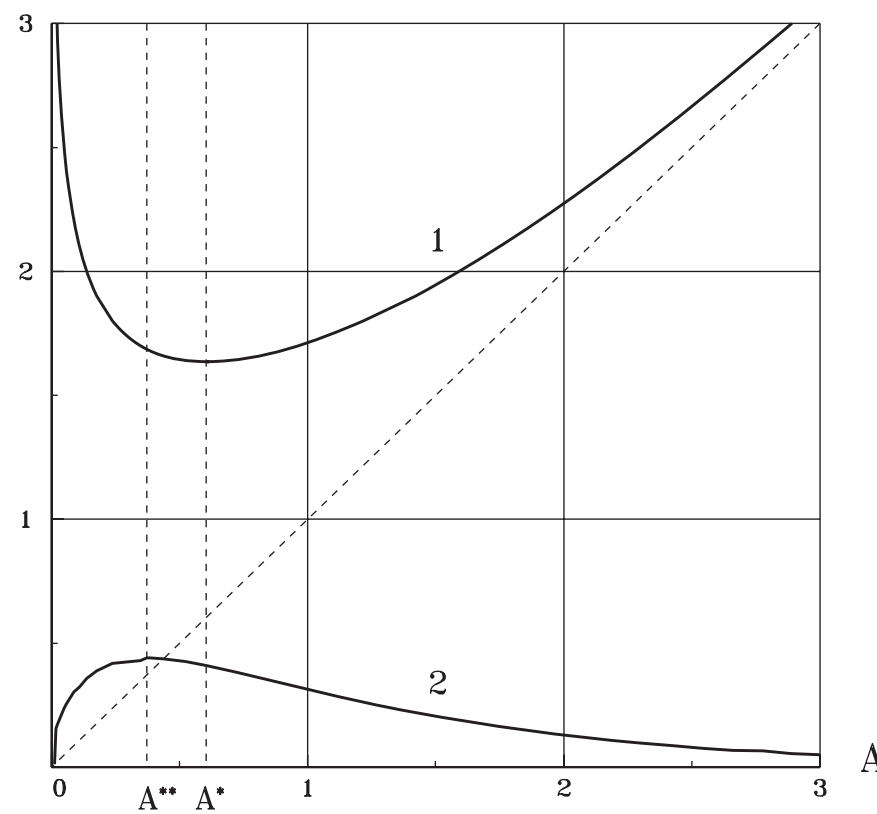

Figure 2. 1-the graphic solution of dispersion equation (14), and 2-angular velocity (15) for the colinear vortex structure, with $\Omega=4 \pi \omega$ (see the value of $\omega$ in the text). Vertical dashed lines at $A=A^{*}$ and $A=A^{* *}$ correspond to extrema of both curves. The dashed line $A=B$ is the asymptote of the dispersion relation at large $A$.

- When the dispersion equation

$$
\frac{B^{2}+A^{2}}{2 A B}=(B+A) K_{1}(B+A)+(B-A) K_{1}(B-A)+A K_{1}(2 B)+B K_{1}(2 A)
$$

is satisfied (where $A=\gamma a$ and $B=\gamma b$ ), a colinear configuration of four vortices rotates around the plane center with constant angular velocity

$$
\omega=\frac{\kappa \gamma^{2}}{4 \pi A}\left[\frac{B^{2}+3 A^{2}}{2 A\left(B^{2}-A^{2}\right)}-K_{1}(B+A)-K_{1}(2 A)-K_{1}(B-A)\right] .
$$

In (14), $B=B_{\min }$ at $A=A^{*}=0.6026$, and, asymptotically, $B \sim A$ at $A \gg 1$ and $B \rightarrow \infty$ at $A \ll 1$. In (15), $\omega=\omega_{\max }$ at $A=A^{* *}=0.3726$, and, asymptotically, $\omega \rightarrow 0$ at $A \gg 1$ and at $A \ll 1$.

Relation (14) between $A$ and $B$ and the distribution of angular velocity along the dispersion curve are plotted in figure 2. Numerical experiments show that stable solutions for $B(A)$ and $\Omega(A)$ (where $\Omega=4 \pi \omega$ ) exist only for $A<A^{* *}$. Further theoretical study will be required to explain this specific value of $A$, but a simple physical argument justifying this result is the following: since $M=\left(\kappa / \gamma^{2}\right)\left(A^{2}-B^{2}\right)$, vortex states with $A>A^{* *}$ have the largest angular momentum for a given angular velocity (note that the same angular velocity $\omega$ can be reached for both $A<A^{* *}$ and $A>A^{* *}$ ). Therefore, larger radial displacements from the steady positions are allowed for $A>A^{* *}$, when the vortex array is perturbed, all the more so as $\omega$ then decreases with $A$. Therefore, stability should be minimal in this case. 
Asymptotic regimes for strong and weak stratifications, respectively, can be obtained:

- When $\gamma \ll 1$ and $a, b=O(1)$, equations (7) and (8) give us

$$
\dot{x}_{j}^{\alpha} \sim \frac{\kappa(-1)^{3-\alpha}}{2 \pi} \times \frac{y_{j}^{\alpha}-y_{j}^{3-\alpha}}{\left(r_{j j}^{\alpha(3-\alpha)}\right)^{2}}, \quad \dot{y}_{j}^{\alpha} \sim \frac{\kappa(-1)^{2-\alpha}}{2 \pi} \times \frac{x_{j}^{\alpha}-x_{j}^{3-\alpha}}{\left(r_{j j}^{\alpha(3-\alpha)}\right)^{2}} .
$$

Obviously, only intra-layer interactions take place then. With our choice of initial conditions, vortices translate as horizontal pairs along the $y$-axis, in opposite directions for the upper and lower layers; their velocities are

$$
\dot{y}_{1}^{1}=\dot{y}_{1}^{2}=-\dot{y}_{2}^{1}=-\dot{y}_{2}^{2}=-\frac{\kappa}{2 \pi(a+b)} .
$$

- When $\gamma \gg 1$ and $a, b=O(1)$, we have

$$
\begin{aligned}
& \dot{x}_{j}^{\alpha} \sim-\frac{\kappa}{4 \pi}\left[(-1)^{3-\alpha} \frac{y_{j}^{\alpha}-y_{j}^{3-\alpha}}{\left(r_{j j}^{\alpha(3-\alpha)}\right)^{2}}+\sum_{\beta=1}^{2}(-1)^{3-\beta} \frac{y_{j}^{\alpha}-y_{3-j}^{\beta}}{\left(r_{j(3-j)}^{\alpha \beta}\right)^{2}}\right], \\
& \dot{y}_{j}^{\alpha} \sim \frac{\kappa}{4 \pi}\left[(-1)^{3-\alpha} \frac{x_{j}^{\alpha}-x_{j}^{3-\alpha}}{\left(r_{j j}^{\alpha(3-\alpha)}\right)^{2}}+\sum_{\beta=1}^{2}(-1)^{3-\beta} \frac{x_{j}^{\alpha}-x_{3-j}^{\beta}}{\left(r_{j(3-j)}^{\alpha \beta}\right)^{2}}\right],
\end{aligned}
$$

and then the interaction between opposite-layer vortices is of 'barotropic' character.

\subsection{Point vortex trajectories in the general case}

A complete analysis of the symmetrical point vortex system can be conducted in terms of the trilinear coordinates $t_{1}, t_{2}, t_{3}$ such that $t_{1}+t_{2}+t_{3}=3$, where

$$
t_{1}=\frac{3 \kappa}{M}\left(r_{12}^{11}\right)^{2}, \quad t_{2}=-\frac{3 \kappa}{M}\left(r_{12}^{21}\right)^{2}, \quad t_{3}=-\frac{3 \kappa}{M}\left(r_{11}^{12}\right)^{2} .
$$

The Hamiltonian can be expressed in these coordinates, within the 'physical region', where the triangle inequality for distances $r_{12}^{11}, r_{12}^{21}$ and $r_{11}^{12}$ is fulfilled. In terms of $t_{j}$, the 'physical region' is defined by

$$
\left(t_{1}\right)^{2}+\left(t_{2}\right)^{2}+\left(t_{3}\right)^{2} \leqslant 2\left(t_{1} t_{2}-t_{1} t_{3}-t_{2} t_{3}\right)
$$

or, after simple transformations,

$$
t_{2} t_{3} \leqslant 9 / 4
$$

Coordinates $t_{1}, t_{2}$ and $t_{3}$ are counted along unit vectors $\mathbf{t}_{\mathbf{1}}, \mathbf{t}_{2}$ and $\mathbf{t}_{3}$ normal to legs of an equilateral triangle with height equal to 3 (see figure 3 ).

In figure 3 , isolines of the Hamiltonian are plotted in the reference frame of the $t_{j}$; this is the phase portrait of the point vortex system (energy must be conserved along trajectories). For any given triplet of these coordinates, the Hamiltonian depends nevertheless on $\gamma$.

For weakly coupled layers $(\gamma=0.1)$, intra-layer vortex interactions are dominant and vortices will couple horizontally. Indeed, we find that vortices pair as horizontal dipoles that move along lines of constant $t_{3}$ (see figure 3(a); horizontal dipoles are called HD below); this regime of horizontal dipole scattering $\left(\begin{array}{l}1 \\ 1\end{array}\right)\left(\begin{array}{l}2 \\ 1\end{array}\right)$ and $\left(\begin{array}{l}1 \\ 2\end{array}\right)\left(\begin{array}{l}2 \\ 2\end{array}\right)$ is labeled $\{1\}$ in this figure. 


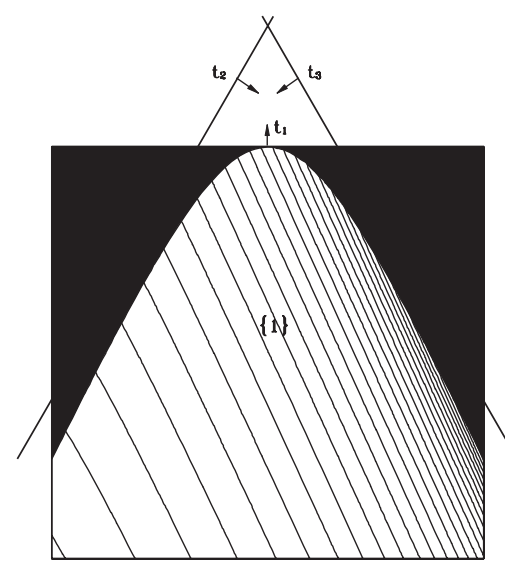

(a)

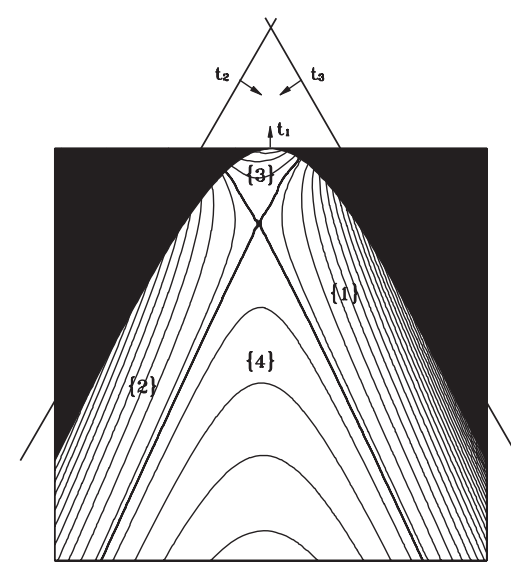

(c)

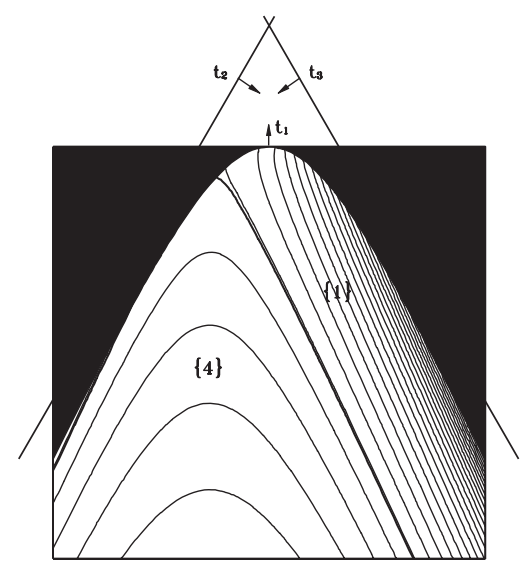

(b)

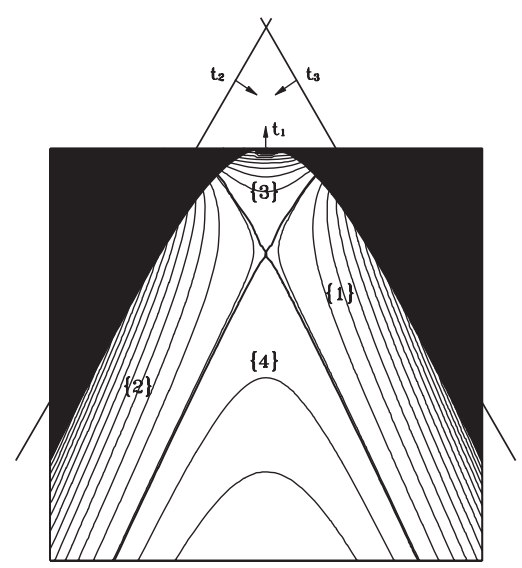

(d)

Figure 3. Phase portraits of the four-vortex system in the $\left(t_{1}, t_{2}, t_{3}\right)$ trilinear coordinate system for $M=-4$ and (a) $\gamma=0.1$, (b) $\gamma=0.5$, (c) $\gamma=1.0$ and (d) $\gamma=4.0$. The 'non-physical' parts of the phase plane are blackened. Thick lines represent separatrices, which separate the different types of vortex interactions. The four regimes are detailed in the text.

For medium-strength layer coupling $(\gamma=0.5)$, inter-layer interactions gain efficiency. If initially $t_{3}$ is relatively large (i.e. for horizontally distant vortices), a new regime appears where a transition between horizontal dipoles and vertically tilted dipoles (called TD) can occur along a trajectory (see figure 3(b)). This regime of vortex scattering via non-aligned pairs, labeled $\{4\}$, can be schematized as

$$
\left(\begin{array}{l}
1 \\
1
\end{array}\right)\left(\begin{array}{l}
2 \\
1
\end{array}\right)+\left(\begin{array}{l}
1 \\
2
\end{array}\right)\left(\begin{array}{l}
2 \\
2
\end{array}\right) \longrightarrow\left(\begin{array}{l}
1 \\
1
\end{array}\right)\left(\begin{array}{l}
2 \\
2
\end{array}\right)+\left(\begin{array}{l}
2 \\
1
\end{array}\right)\left(\begin{array}{l}
1 \\
2
\end{array}\right)
$$

Note that vortex configurations for motions of this type do not contain a colinear structure, and therefore cannot be characterized by initial conditions (11). Motions of type $\{4\}$ are illustrated in figure 4. 


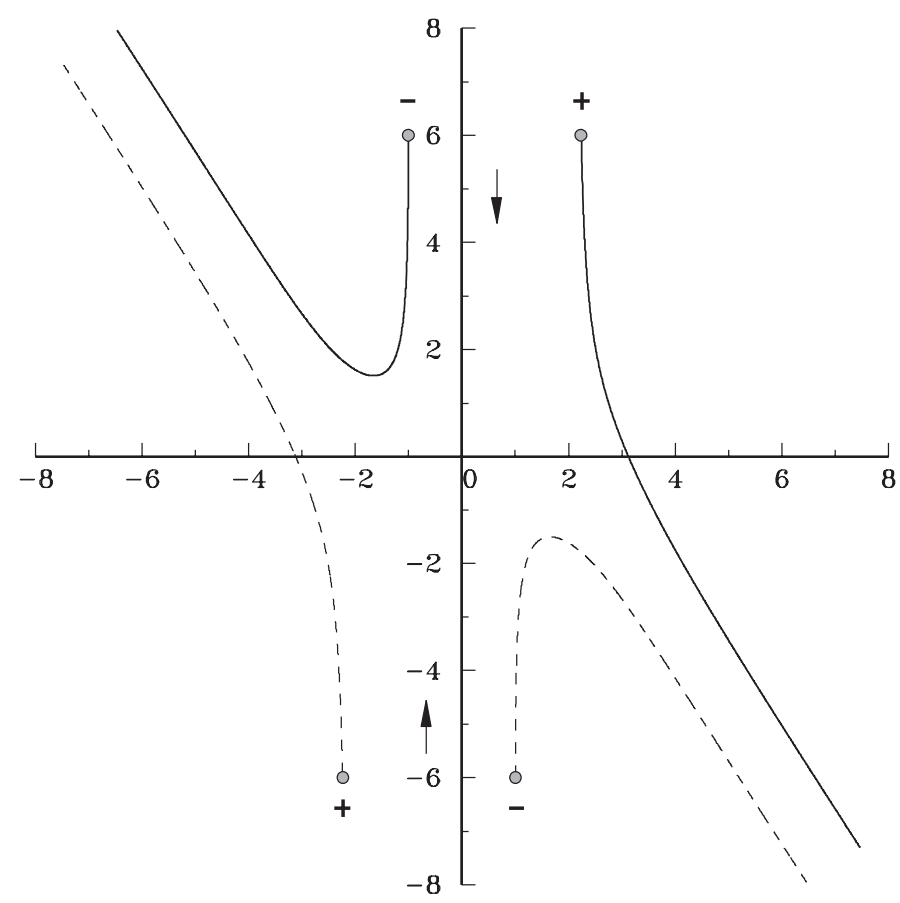

Figure 4. Vortex trajectories for motions of type $\{4\}$ with $\gamma=0.5$ and $M=-4$. The initial positions of the vortices are marked by circles. Here and in all other figures displaying vortex evolutions, polarities are indicated near each vortex at the initial moment. Arrows indicate the direction of initial motion of vortices. Here and in figures 5-8, solid (dashed) lines correspond to upper- (lower-) layer vortex trajectories.

When the layers are strongly coupled ( $\gamma=1.0$ or $\gamma=4.0$ ), inter-layer vortex interactions become dominant and vertically coupled vortex structures prevail. Then, two regimes appear:

- One where the vertically tilted dipoles remain coupled all along their trajectories (see figure $3(\mathrm{c})$ ). This regime of vortex scattering via hetons $\left(\begin{array}{l}1 \\ 1\end{array}\right)\left(\begin{array}{l}2 \\ 2\end{array}\right)$ and $\left(\begin{array}{l}2 \\ 1\end{array}\right)\left(\begin{array}{l}1 \\ 2\end{array}\right)$ is labeled $\{2\}$ in figure 3.

- One where all vortex trajectories are closed, contrary to the previous regimes (see figure 3(d)). This regime is labeled $\{3\}$ in figure 3 . In this regime, vortices form antihetons $\left(\begin{array}{l}1 \\ 1\end{array}\right)\left(\begin{array}{l}1 \\ 2\end{array}\right)$ and $\left(\begin{array}{l}2 \\ 1\end{array}\right)\left(\begin{array}{l}2 \\ 2\end{array}\right)$, and follow trajectories inside a bounded subdomain, with different average angular velocities. However, at the stationary elliptic point, on the boundary of this region, condition (14) is fulfilled, and the vortex structure is colinear, in solid body rotation at constant angular velocity (15). In this regime, $t_{1}$ (the distance between likesigned vortices in opposite layers) varies little - all the less so as layer coupling is intense. For the largest values of $\gamma$, trajectories in regime $\{3\}$ become more symmetrical in $t_{2}$ and $t_{3}$.

How this phase space description translates in physical space is now illustrated with trajectories of point vortices.

In figure 5, trajectories of types $\{1\}$ and $\{3\}$ are shown for $a=0, b=2$ and for increasing $\gamma$. Initially, all vortices are located along the $x$-axis, and the central ones, $\left(\begin{array}{l}1 \\ 1\end{array}\right)$ and $\left(\begin{array}{l}1 \\ 2\end{array}\right)$, represent an aligned antiheton with positive vortex intensities; the peripheral anticyclonic vortices $\left(\begin{array}{l}2 \\ 1\end{array}\right)$ and $\left(\begin{array}{l}1 \\ 2\end{array}\right)$ are located on the left-hand side and right-hand side, respectively. 


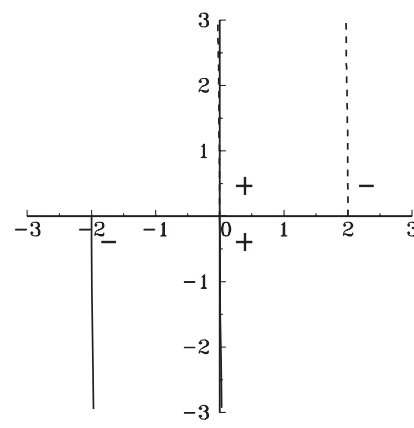

(a)

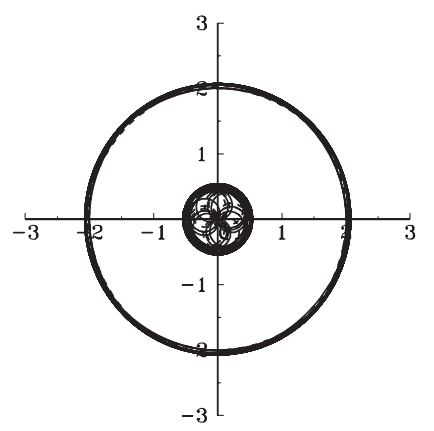

(d)

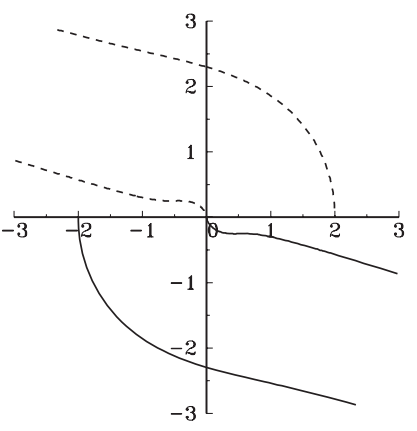

(b)

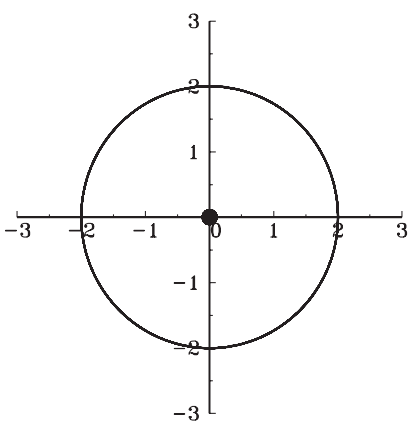

(e)

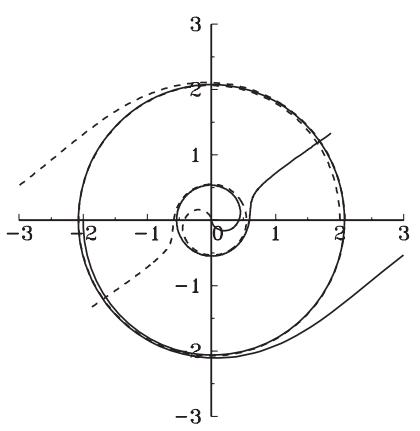

(c)

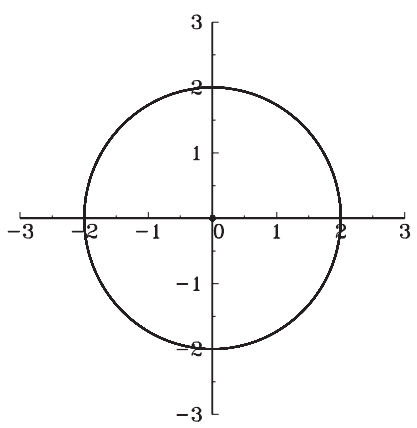

(f)

Figure 5. Examples of vortex trajectories of type $\{1\}$ - panels (a), (b), (c)—and of type $\{3\}$ panels (d), (e), (f). All initial states are colinear ones, and parameters are $a=0, b=2, M=-4$ and (a) $\gamma=0.1$, (b) $\gamma=0.9$, (c) $\gamma=0.95659$, (d) $\gamma=0.95660$, (e) $\gamma=1.2$ and (f) $\gamma=1.5$. Here and in figures $6-8$, the intensities of the point vortices coincide with those shown in figure 1 .

Clearly, for the same initial positions, there exists a critical value of stratification (here $\gamma=0.95659$ ) for which trajectories change from open to closed (i.e. from regime $\{1\}$ to regime $\{3\}$ ). This is explained physically by the increasing influence of co-rotation (via layer coupling) against translation (simple horizontal vortex coupling). Indeed, with the help of the analytical formulae of asymptotic regimes (see the end of the previous subsection), one notices that for small $\gamma$, the motion is translation along the $y$-axis, whereas for large $\gamma$, layer coupling will induce a component of rotation in the motion of the vortex array. In between, due to the conjugate effect of rotation and translation, the central vortices exhibit a complex trajectory, which is quasi-periodic. The spatial domain occupied by this trajectory shrinks with increasing $\gamma$.

Now, we detail this complex trajectory, and study its sensitivity to the initial position of the central vortices. Figure 6 presents the trajectories, both in a fixed and in a rotating frame of reference, of the vortex array for $\gamma=1, a=0$ and $a=-0.04$ (motions of type $\{3\}$ ). Due to time periodicity of the motion in the rotating frame of reference, complex trajectories in the fixed frame of reference can correspond to simple trajectories in the rotating frame of reference (the latter are also called 'choreography'; see Borisov and Mamaev 2005). Quasiperiodicity or aperiodicity depends, in particular, on parameter $a$. Values of $a$ corresponding to stationary colinear vortex configurations can also be found, as will be shown now.

A scenario for the transition between a colinear stationary structure and non-colinear structures is presented in figure 7. For the colinear structure (figure 7(a)), the dispersion 

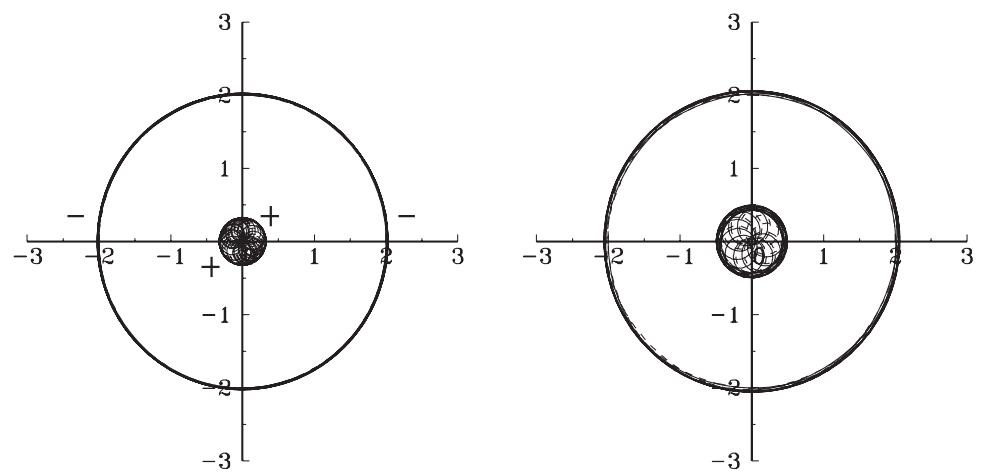

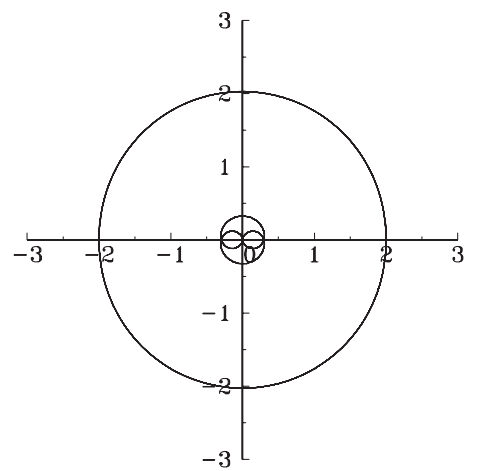

(a)

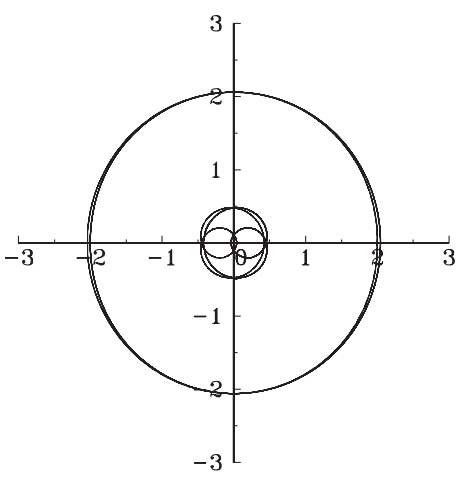

(b)

Figure 6. Vortex trajectories in a fixed frame of reference (1) and in a rotating frame of reference (2) for motions of type $\{3\}$ with $\gamma=1.0, M=-4$ and (a) $a=0$ and (b) $a=-0.04$. Angular velocities for rotating frames are respectively $\omega=-0.0000164$ and $\omega=-0.00001665$.

equation (11) is satisfied; in the four other cases (figures 7(b)-(e)), the dispersion equation (11) has a small discrepancy. Here only the initial stage of vortex motion is illustrated. The positions of the vortices at the initial and final (calculated) moments are linked by segments. In the numerical experiments shown in figures 7(b)-(e), the disturbance of the stationary configuration is obtained by changing parameter $a$ from its equilibrium value $a^{*}=0.346$, while other parameters are kept constant. A variation $\Delta a=0.023$ of distance $a$ is chosen for the following reasons:

- At $a=a^{*}+\Delta a$ (figure 7(b)) and at $a=a^{*}-\Delta a$ (figure 7(d)), phase trajectories still belong to regime $\{3\}$ of closed type.

- However, at $a=a^{*}+2 \Delta a$ (figure 7(c)) and at $a=a^{*}-2 \Delta a$ (figure 7(e)), phase trajectories are already located in regime $\{2\}$ in the first case and in regime $\{1\}$ in the second case.

It is easy to see that with growing $a$, colinearity is violated because internal vortices begin to move ahead of the external ones at the very first stage of the evolution (figure 7(b)). This provides the conditions for shifting regime from the colinear vortex array to tilted point hetons. Conversely, when $a$ decreases, the external vortices run ahead (figure 7(d)), leading to a transition to the regime of horizontally coupled vortices.

Figure 8 illustrates this transition even further. Vortex trajectories for the motion of type $\{3\}$ are shown in figures $8(\mathrm{~b})-(\mathrm{d})$; the central one is the stationary colinear state, and the 

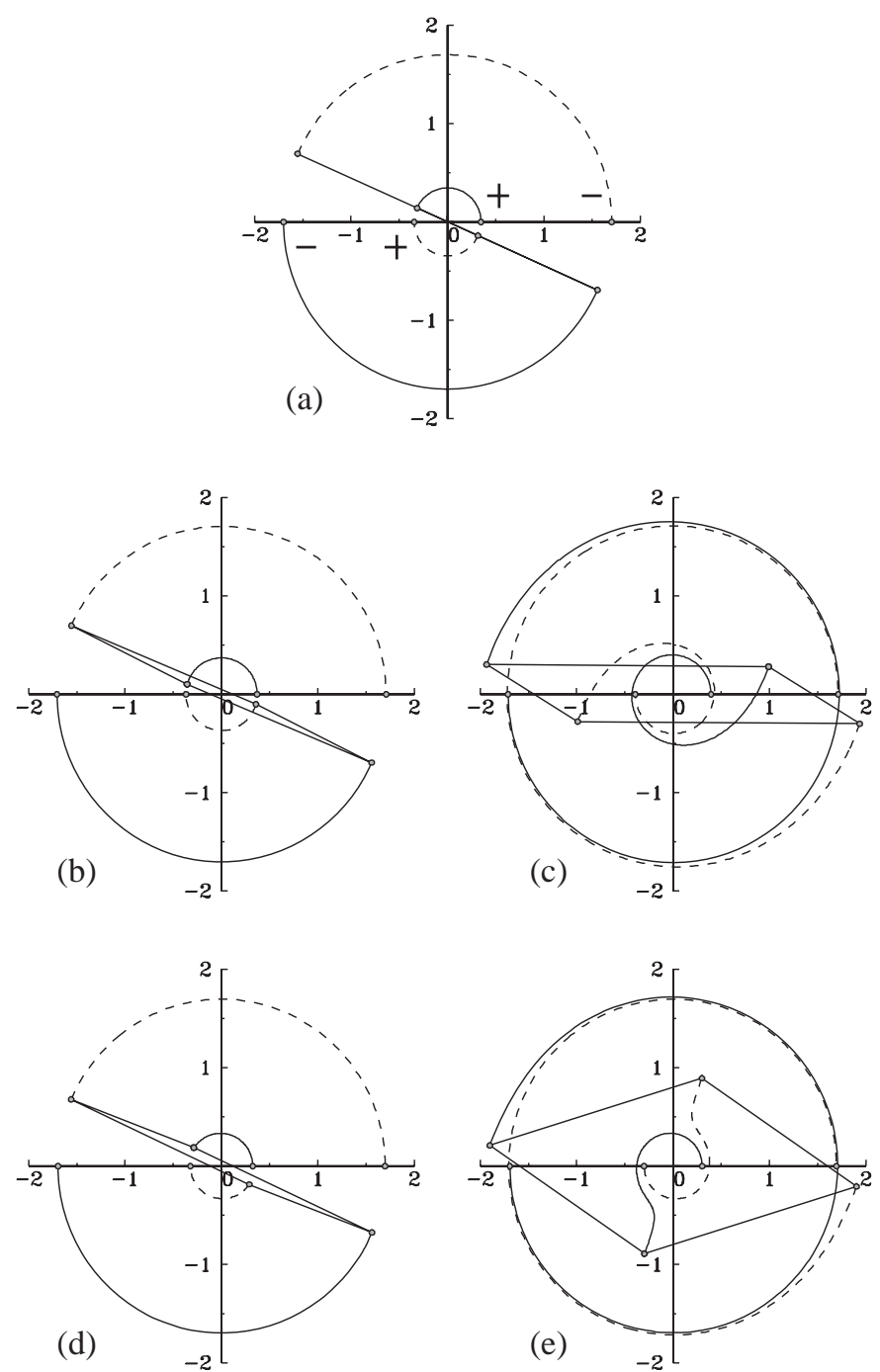

Figure 7. Transition between a colinear stationary structure and two horizontal dipoles or two tilted dipoles (hetons) for $\gamma=1, M=-2.7703$ and (a) $a=0.346$, (b) $a=0.369$, (c) $a=0.392$, (d) $a=0.323$ and (e) $a=0.300$.

lateral ones are the disturbed stationary states, which correspond nearly to the limit values of the parameter $a$ inside domain $\{3\}$. For each numerical experiment, the vortex configuration performs 50 cycles with respect to the center of vorticity. Figures 8(a) and (e) display the trajectories of types $\{1\}$ and $\{2\}$, respectively. The values of $a$ for the last two cases are also located near the domain boundaries of the corresponding solutions. Before entering a scattering regime, the vortices have performed more than two cycles with respect to the center of vorticity. Thus, we may conclude that at the given value of angular momentum $M=$ -2.7703 an approximate interval $a \in(0.323 ; 0.370)$ corresponds to bounded trajectories.

Let us finally note the following:

- The stationary state (figure $8(\mathrm{c})$ ) corresponds to a motionless elliptical point in area $\{3\}$ of the phase portrait. 

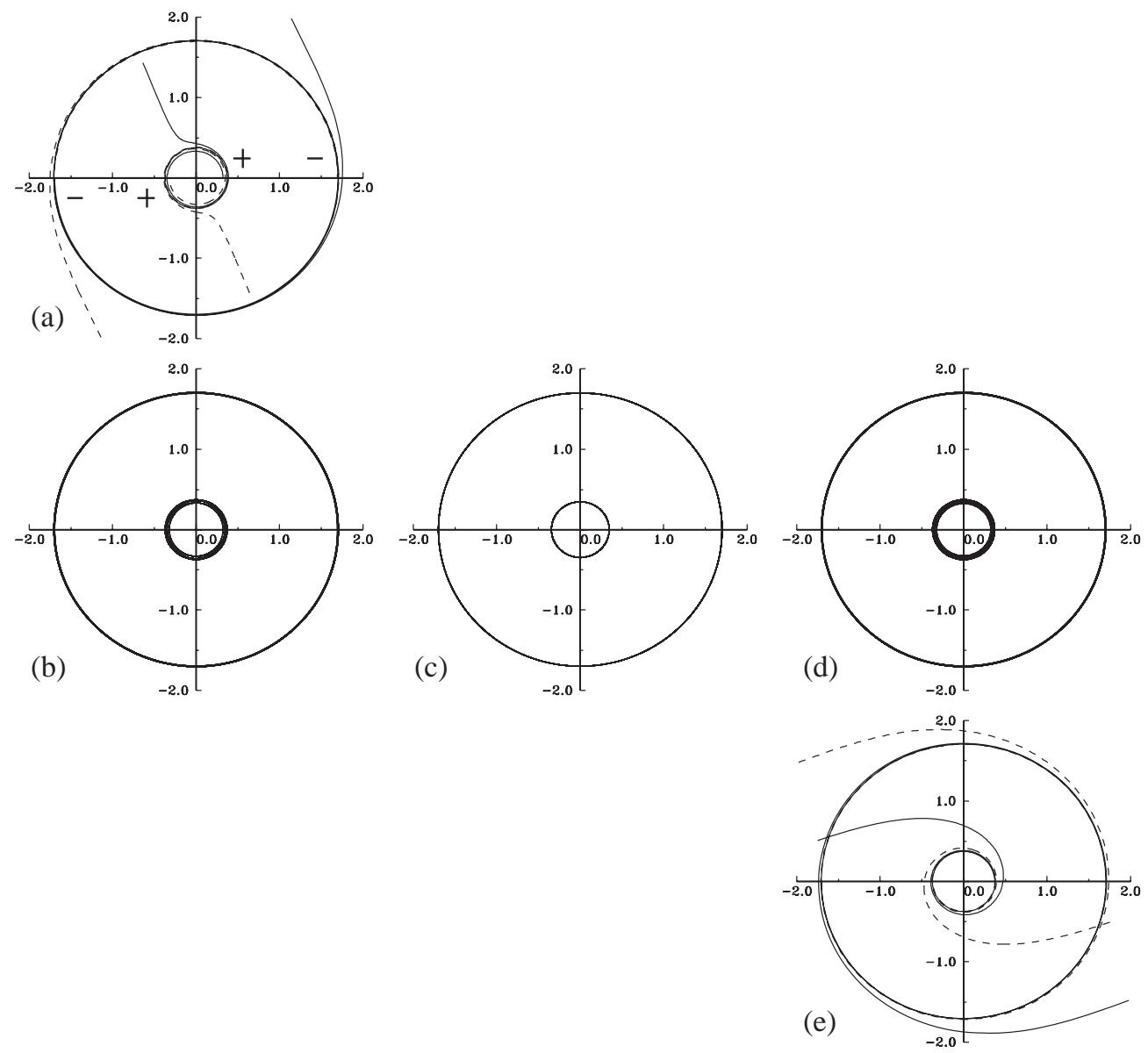

Figure 8. Same as figure 7, but for longer computing time and for (a) $a=0.322$ (analogue of figure $7(\mathrm{e}$ ), type $\{1\}$ ), (b) $a=0.323$ (case of figure $7(\mathrm{~d})$, type $\{3\}$ ), (c) $a=0.346$ (case of figure 7(a), type $\{3\}$, colinear state), (d) $a=0.370$ (analogue of figure 7(b), type $\{3\}$ ) and (e) $a=0.371$ (analogue of figure 7(c), type $\{2\}$ ).

- The initial configurations (figures 8(b) and (d)) correspond to points close to the crossing of the boundary of area $\{3\}$ and of the separatrices (to the right and to the left of the elliptical point, respectively).

- The initial state of figure 8(a) corresponds to a point on the boundary of area $\{1\}$, and the initial state of figure 8 (e) to a point on the boundary of area $\{2\}$; both of them are also located in the vicinity of the separatrix.

\section{Nonlinear evolutions of finite-area vortices}

Now we evaluate the influence of a finite vortex size on the evolutions of an opposite-signed heton pair. A contour surgery code (see section 2) is initialized with finite-area vortices having strengths (area-integrated potential vorticity) equal to those of point vortices, but with unit radius. Finite-area vortices, in contrast to point vortices, are able to merge (if they are likesigned and initially close to each other) or to decay into smaller structures (when unstable or strongly sheared). Moreover, both processes are usually accompanied by filamentation. 
b

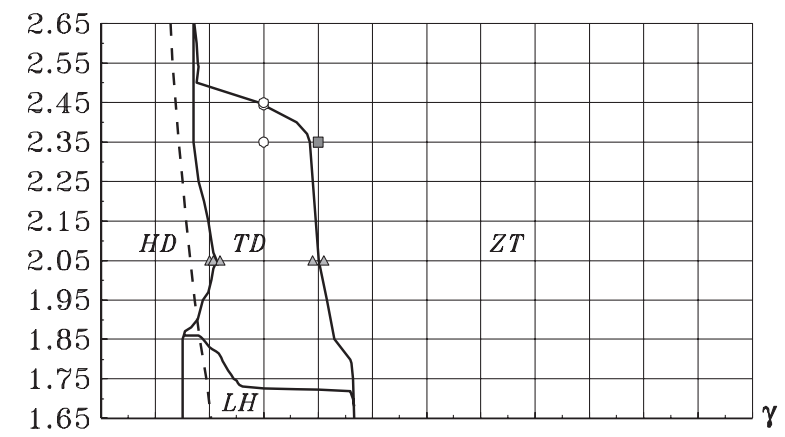

b

(b)

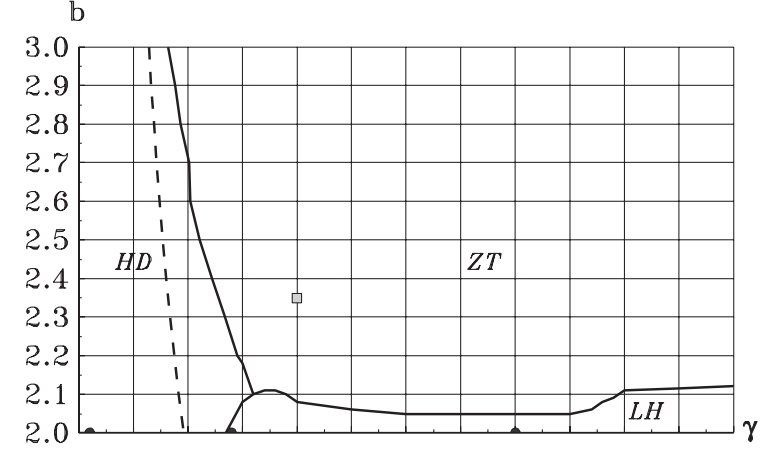

b

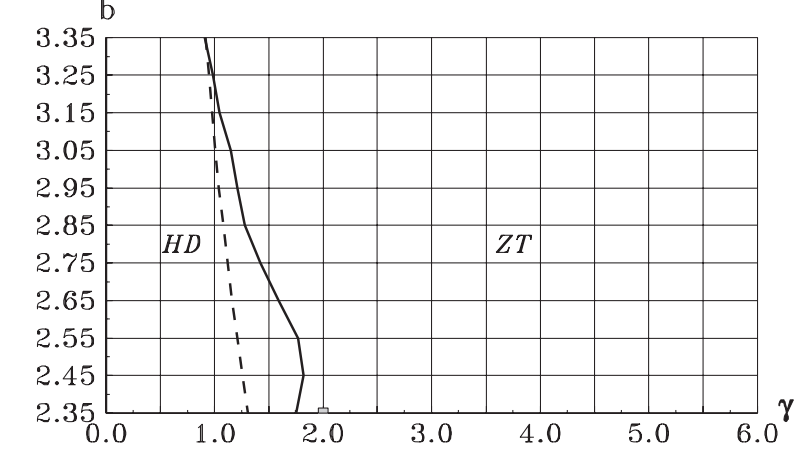

Figure 9. Regime diagrams for the finite-area heton pair in the $(\gamma, b)$ plane for (a) $a=0.35$, (b) $a=0$ and (c) $a=-0.35$. Notations: HD, two horizontal dipoles (regime $\{1\}$ ); TD, two vertically tilted dipoles (regime $\{2\}$ ); LH, two L-shaped hetons; ZT, Z-shaped tripole (regime $\{3\}$ ). Dashed lines represent the boundaries between regions $\{1\}$ (left) and $\{3\}$ (right) for the point heton pairs. These boundaries were determined numerically with the point vortex model. They are related to the separatrices of figure 3 , but in a different parameter plane. The meaning of the markers is explained in the captions of figures 10,11, 13 and 14.

When describing the results of numerical experiments with finite-area vortices, nondimensional units of time are used everywhere. The non-dimensional time unit is the rotational period of a fluid particle on a unit radius contour, for a heton with vertical axis, for each $\gamma$.

Figure 9 presents a regime diagram in parameter space, summarizing the results of many numerical simulations: it indicates the final state of the vortex system, as $(a, b, \gamma)$ are varied. 
Table 1. Characteristics of baroclinic vortices described in the text and figures. The domains of formation are given for $a=0$ for both colinear initial configuration and head-on collisions.

\begin{tabular}{|c|c|c|}
\hline Acronym & Type of vortex structure & Domain of formation \\
\hline HD & Horizontal dipole & Weak layer coupling \\
\hline LH & $\begin{array}{l}\text { L-shaped dipole (a columnar vortex } \\
\text { associated with a satellite in one layer) }\end{array}$ & $\begin{array}{l}\text { Medium layer coupling and small to } \\
\text { medium distance between vortices }\end{array}$ \\
\hline TD & Vertically tilted dipole & $\begin{array}{l}\text { Medium to strong layer coupling and } \\
\text { medium distance between vortices }\end{array}$ \\
\hline ZT & $\begin{array}{l}\text { Z-shaped tripole (a columnar vortex } \\
\text { with one satellite in each layer) }\end{array}$ & Strong layer coupling \\
\hline UZT & Unstable Z-shaped tripole & $\begin{array}{l}\text { Strong layer coupling and } \\
\text { large distance between vortices }\end{array}$ \\
\hline
\end{tabular}

All calculations share similar features in their initial conditions:

- Vortices are circles of unit radius in a colinear configuration.

- Their centers are located on the $x$-axis.

- The distances between them are given by parameters $a$ and $b$ (see figure 1).

In figures $9(\mathrm{a})-(\mathrm{c})$, the minimum value of $b$ corresponds to contact between same-layer vortex contours.

The abbreviations for the various nonlinear regimes shown in figure 9 are the following:

- HD is the formation of two scattering 'horizontal' dipoles (analogue of motions of type $\{1\}$ for point vortices).

- TD is the formation of two vertically 'tilted' dipoles (analogue of motions of type $\{2\}$ for point vortices).

- LH is the formation of an L-shaped dipole, composed of a like-signed vortex column, associated with an opposite-signed vortex in one layer (the segments linking the centers of the vortex patches form a letter L, which may be normal or inverse); this vortex array does not have a pointwise equivalent.

- ZT is the formation of a Z-shaped tripole, composed of a like-signed vortex column, associated with an opposite-signed vortex in both layers, but lying at symmetric positions with respect to the central vortex. The segments linking the centers of vortex patches look like the letter $\mathrm{Z}$. This vortex array has a pointwise equivalent only for very large values of $\gamma$.

In figure 17 , another regime, UZT, is mentioned; it corresponds to an unstable Z-shaped tripole (see also table 1 ).

Firstly, figure 9 shows that the various regimes depend on all three parameters, but stratification clearly has a dominant influence. Indeed, as for point vortices, motion of type $\{1\}$ occurs for weak layer coupling $\gamma$ and motion of type $\{3\}$ for moderate or strong layer coupling predominantly. This is why we first investigate the influence of parameter $\gamma$ on finite-area heton interaction.

Secondly, this figure shows that parameter $b$ is less influent, except for $a \geqslant 0$ where regime transitions ( $\mathrm{LH}$ to $\mathrm{TD}$ or $\mathrm{ZT}$ ) occur. The HD regime does not exhibit a notable sensitivity to parameter $b$. Thus, we will investigate the dependence of nonlinear regimes on the geometrical parameters in a second stage.

Finally, the transition between the regimes of horizontal dipoles and of compact vortex compounds (the equivalent of Z-shaped tripoles) for point vortices is indicated in figure 9. This comparison shows the similarity between point vortex dynamics and finite-area vortex 
(a)

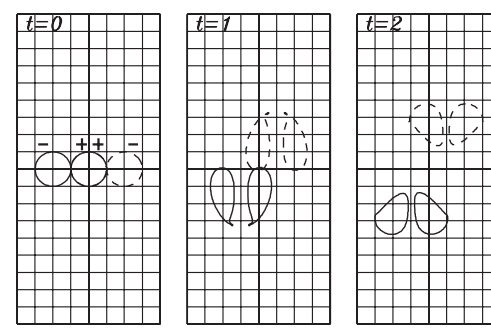

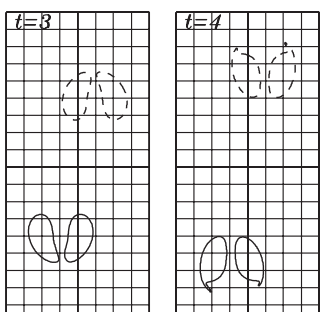
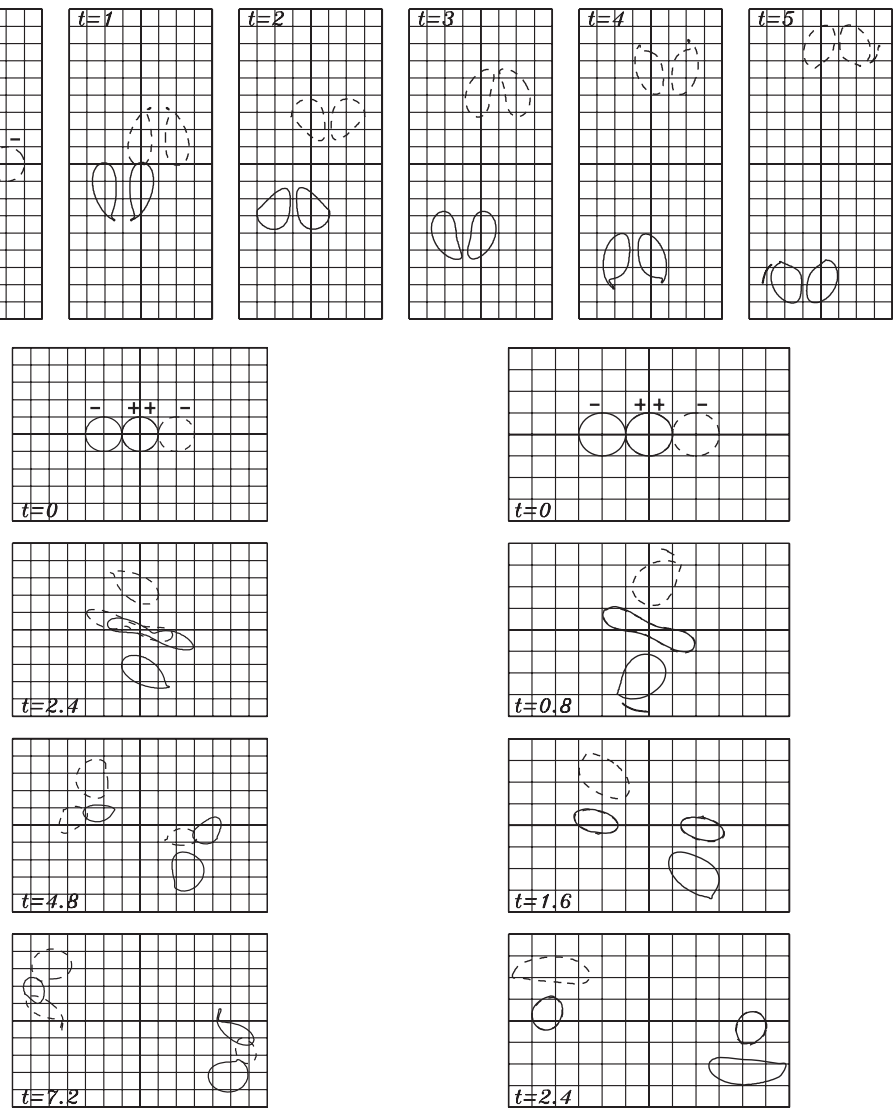

(b)
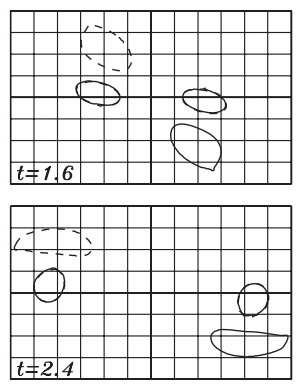

(c)

Figure 10. Contour surgery simulations (CSS) of the nonlinear evolution of finite-area heton pairs with $a=0, b=2$ and (a) $\gamma=0.1$ (cf figure 5(a)), (b) $\gamma=1.4$ and (c) $\gamma=4.0$ (circle markers in figure 9(b)). Here and in all figures with CSS, except figure 12, solid (dashed) lines correspond to upper- (lower-) layer vortex contours. The polarity of the finite-area vortices (here and in figures 11-15) coincides with that shown in figure 1.

dynamics, as long as vortex splitting and merging (specific of finite-area vortices) are not involved. Indeed, figure 9(a) indicates a transition between regimes HD and TD for finitearea vortices, but no transition between point vortex regimes $\{2\}$ and $\{3\}$ for the values of $(\gamma, a, b)$ in this case. As will be shown in section 4.1.2, a transitional state, with substantial deformation of the central vortex, exists when interacting finite-area hetons form an HD for $a>0$. Since point vortices do not possess internal degrees of freedom, they cannot form an HD structure in this case.

\subsection{Influence of layer coupling}

4.1.1. Initially aligned central cyclones. We first study the case of central cyclones initially aligned vertically $(a=0)$.

For weak layer coupling $(\gamma=0.1)$, the nonlinear evolution leads to horizontal dipoles HD (see figure 10(a)). This evolution presents a striking similarity to that of point vortices (figure 5); indeed, opposite-layer contributions in equations (7) and (8) vanish as $\gamma \rightarrow 0$. 
Once formed, horizontal dipoles drift away in opposite directions along the $y$-axis. The mutual deformation induced on each vortex contour by the others is mainly composed of azimuthal modes 1 and 2; this contour perturbation continues to oscillate on each traveling dipole, but it decreases in amplitude every time that vorticity crosses the separatrix and filaments (see time $t=5)$.

For medium to strong layer coupling and for close peripheral vortices $(\gamma>1.3$ and $b \leqslant 2.1$ ), L-shaped hetons (LH) are formed-a newly observed vortex compound that has no pointwise equivalent in our study (see figure $9(\mathrm{~b})$ ). Note that this evolution is observed more generally when the central cyclones overlap significantly.

In figure 10(b), this LH formation regime is illustrated for $\gamma=1.4$. Owing to the strong shear due to opposite-layer anticyclones, the central cyclones split and each anticyclone pairs with half the cyclone in its layer and half the cyclone in the opposite layer. L-shaped dipoles then propagate in opposite directions in the two layers. In each L-shaped structure, the internal antiheton vacillates, interacting with the anticyclone. If $\gamma$ still increases, the two layerwise cyclones become more coupled and the tilt of the internal antiheton decreases (see figure 10(c) for $\gamma=4.0)$.

The transition from $\mathrm{HD}$ to $\mathrm{LH}$ formations is due to the larger inter-layer vortex interaction, which strengthens the shear exerted on the central vortices.

For medium to strong layer coupling and for distant peripheral vortices $(b>2.1$ and $\gamma>\gamma_{\mathrm{c}}(b) \sim 0.8-1.5$, the two-heton interaction forms a Z-shaped tripole (ZT). This finitearea vortex compound is also a newly observed baroclinic multipole. It will be described in more detail in the following subsection for $a \neq 0$ and in section 4.2 for $a=0$.

The transition from LH to ZT formations is due to the weakening influence of peripheral vortices, and therefore to a decreased shear on the central vortex, which remains stable and aligned in ZT.

4.1.2. Initially distant central cyclones. When the central vortices are not aligned, the question arises about their possible realignment in the course of nonlinear vortex interactions. Noting that the case $a=0.35$ provides the largest diversity of regimes, we study the influence of layer coupling $\gamma$ on the heton pair interaction for $b=2.05$ (figure 11). The transition between regimes HD (figure 11(a)) and TD (figure 11(c)) takes place between $\gamma=1.0$ and $\gamma=1.1$. Again it is natural that weaker layer coupling favors horizontal vortex interaction and stronger layer coupling favors vertical vortex interaction.

During the first stage of the evolution, the vortex array evolves toward a Z-shaped tripole (ZT), which, however, is unstable. After a short period (here, about $t=4$ ) it proceeds either to an HD or to a TD, depending on $\gamma$. The transitional state will last all the longer as $\gamma$ is closer to its critical value in the interval $\gamma \in[1.035,1.036]$ (see figure $11(\mathrm{~b})$, where $\gamma=1.035$ ).

As $\gamma$ increases again, the boundary between the TD and ZT regimes is reached. Indeed, as layer coupling increases, the vertical alignment of the like-signed central vortices is rendered more efficient, and Z-shaped tripoles are more likely to form.

For $\gamma=1.95$ (figure 11(d)), the nonlinear regime is of type $\{2\}$, but the central cyclones undergo active filamentation and shed small vortex patches (the area loss of the cyclonic vortices is $29 \%$ ).

For $\gamma=2.05$, a stable Z-shaped tripolar structure is formed (figure 11(e)). This structure is the finite-area equivalent of the end state of type $\{3\}$ for point vortices. Again stabilization is preceded by active filamentation and cyclonic vorticity losses are about $34 \%$.

Figure 12 demonstrates that filament shedding is related to vortex fluid crossing the separatrices. One can clearly see how the vorticity of the central vortices is trapped beyond the saddle point of the co-rotating streamfunction and enters the external recirculation 
(a)

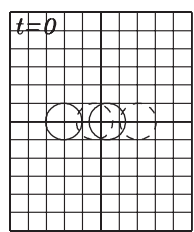

(b)

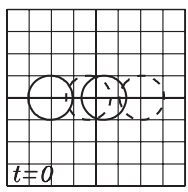

(c)

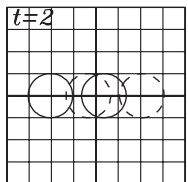

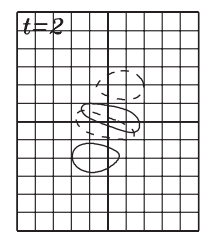
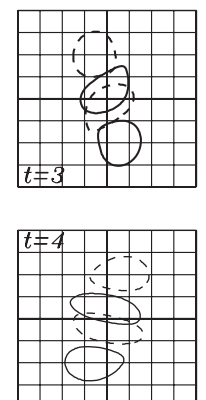
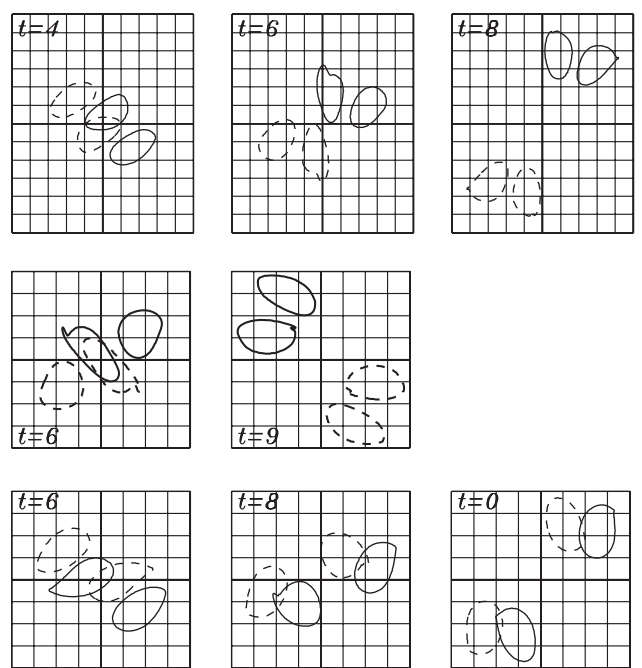

(d)
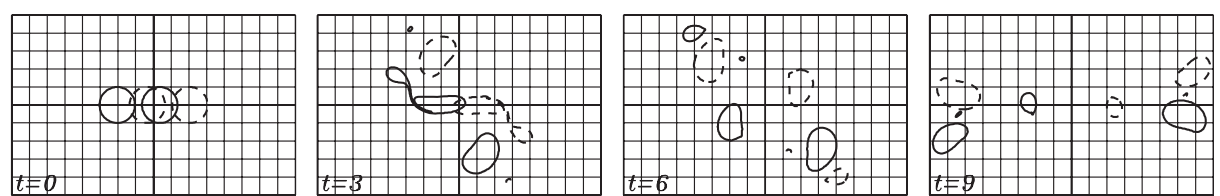

(e)
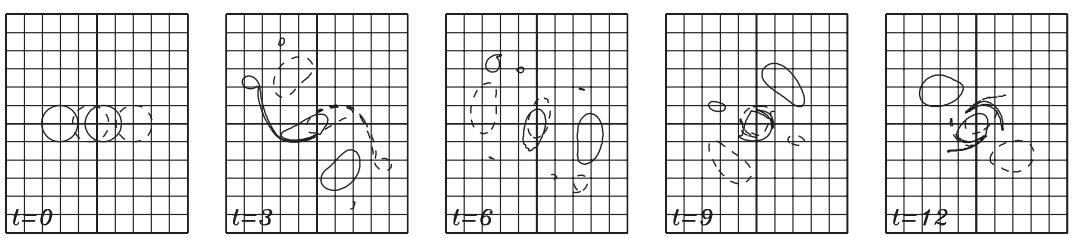

Figure 11. Evolution of finite-area heton pairs for $a=0.35, b=2.05$ and (a) $\gamma=1.0$, (b) $\gamma=1.035$, (c) $\gamma=1.1$, (d) $\gamma=1.95$ and (e) $\gamma=2.05$ (triangle markers in figure 9(a)).

domain. This patch of vorticity expelled by the central vortices forms a smaller vortex that is initially connected to the saddle point area via a filament. This filament extends along the separatrix.

To summarize, for weak layer coupling, the dominant mechanism is intra-layer interaction leading to horizontal dipoles HD, whereas for moderate or strong layer coupling, vertical vortex interaction becomes dominant and the outcome of these interactions is two scattering vortex dipoles (TD), L-shaped hetons (LH) or a rotating Z-shaped tripole.

\subsection{Influence of the initial distance between vortices}

Firstly, we examine the effect of parameter $b$ (distance of the external vortices) on the evolution of hetons at fixed $a=0.35$ and $\gamma=1.5$ (see the regime diagram in figure 9(a)). Physically, we can expect that close peripheral and central vortices will strongly interact and thus form (horizontal or vertical) vortex pairs, while distant peripheral vortices will weakly advect and deform central vortices, resulting in the formation of a tripole. 

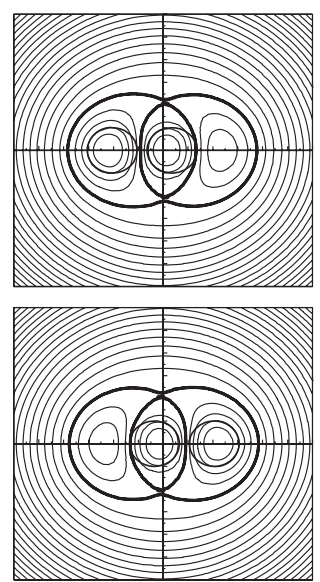

$t=0$
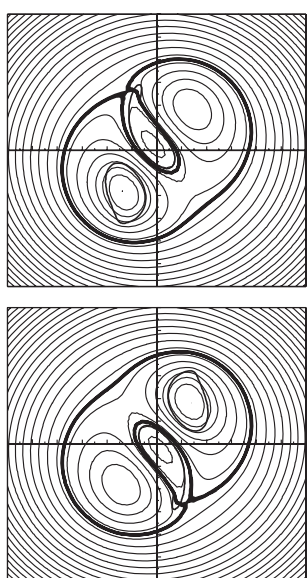

$t=1$
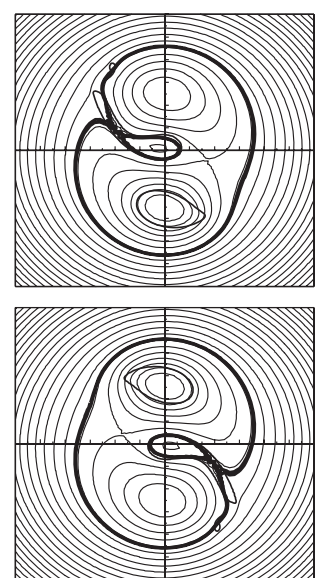

$t=2$
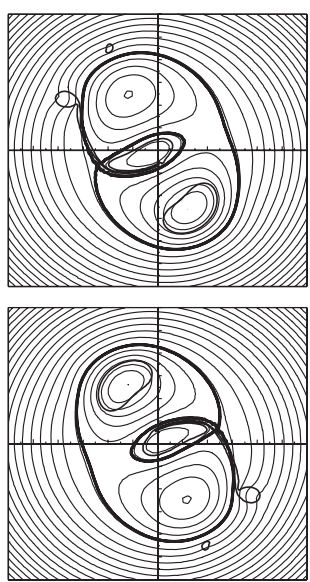

$t=3$

Figure 12. Streamlines in the co-rotating frame and vortex patches in the upper layer (upper line) and in the bottom layer (lower line) at the same early times as figure 11(e). Streamlines are drawn in thin lines, separatrices in thicker solid lines and vortex contours in bold lines.

And indeed, figure 9(a) shows that two regime transitions occur, one at $b=1.72$ between LH and TD and one at $b=2.43$ between TD and ZT. We concentrate here on the transition between dipoles and tripoles.

Figure 13 shows the transition between the TD and ZT regimes. Figures 13(a) and (b) correspond to initial conditions getting closer to the threshold. In the first case, the vortex array rotates approximately by $\pi / 2$ before being scattered, while in the second case, it turns over $3 \pi / 2$. In the second case, the intermediate stage (from $t=2$ to $t=10$ ) evidences a vortex array with centers forming a uniformly rotating diamond. But these arrays are unstable and the two hetons are finally scattered. For the central vortices to become aligned, a larger value of $b$ is necessary. It corresponds to a weaker influence of external vortices. For example, figure 13(c) (corresponding to $b=2.45$ ) evidences the formation of a Z-shaped tripole. During the formation of this tripole (between $t=4$ and $t=8$ ), the vortex centers form a diamond that flattens with time. Furthermore, the internal antiheton, which determines the global rotation, begins to spin faster than the external vortices. As a result, the vortex pulsates and is not stationary, but the whole compound remains a Z-shaped tripole. Note also that little vorticity is lost via filamentation $(3.5 \%)$.

Secondly, we evaluate the influence of parameter $a$ on the type of evolution. Note that TD and LH structures appear only for $a \geqslant 0$. Indeed, inter-layer coupling between opposite-signed vortices will occur if they are initially close enough, and at least closer than opposite-signed vortices in the same layer.

Figure 9 also indicates that, in the $(a, b, \gamma)$ space, the most widespread structures are ZT (Z-shaped tripoles).

Figure 14 shows the influence of varying $a$ on the formation of ZT, at fixed $b$ and $\gamma$. The first two cases (figures 14(a) and (b)) correspond to a vortex permutation $\left(\begin{array}{l}1 \\ 1\end{array}\right)$ and $\left(\begin{array}{l}1 \\ 2\end{array}\right)$ in the initial internal antiheton. Due to the periodicity of the process (neglecting filamentation), the configurations should be interchangeable at times of colinearity. The two evolutions are indeed very similar. Due to the sufficiently strong layer coupling, the central vortices reach an $80 \%$ alignment rate, and a Z-shaped tripole is formed. The small peripheral vortices weakly interact with the four other vortices and do not contribute much to their oscillations. 

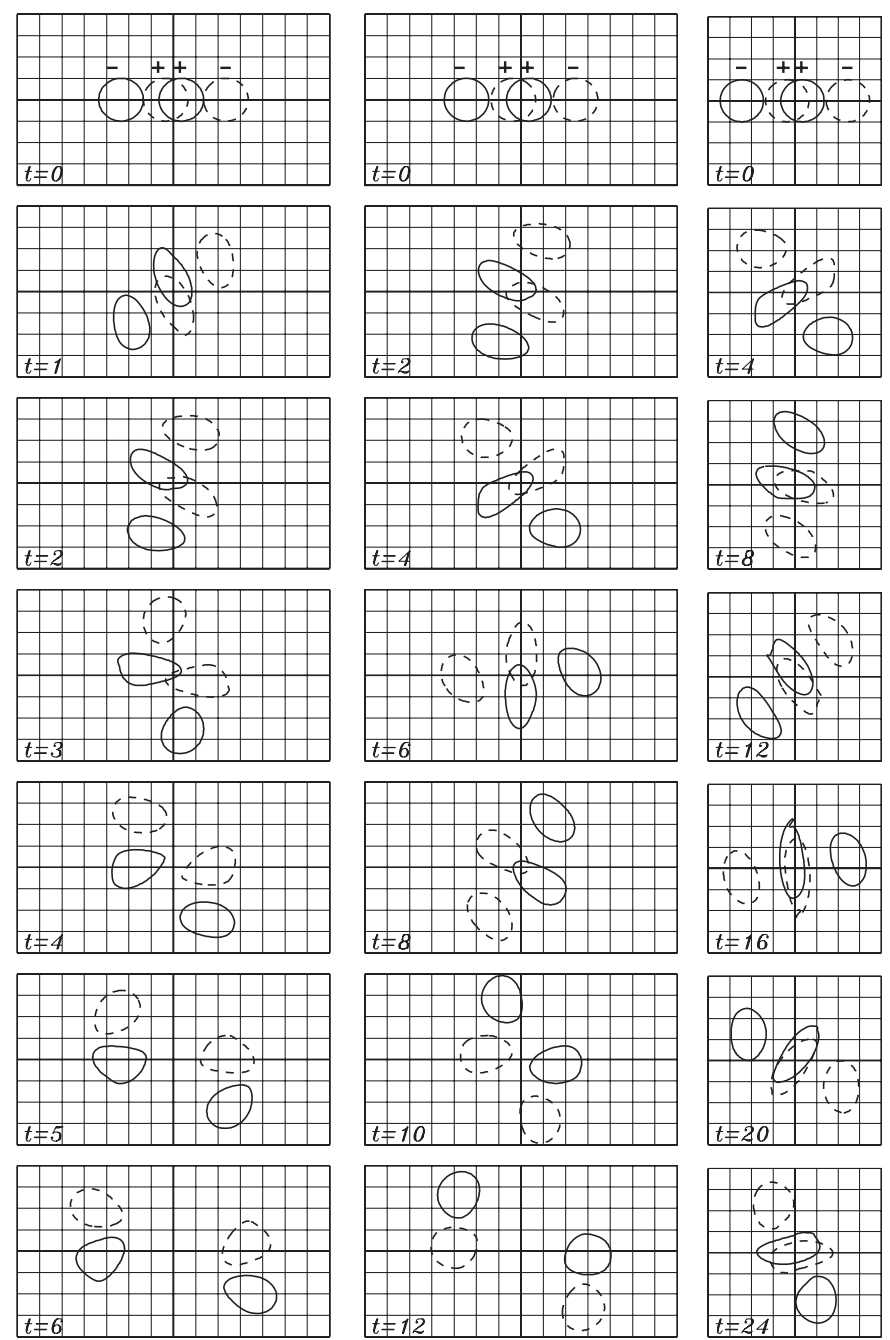

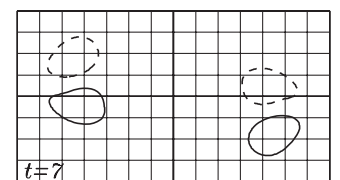

(a)

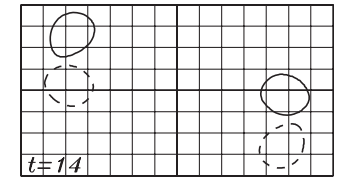

(b)

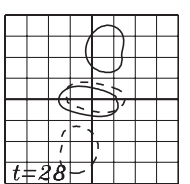

(c)

Figure 13. Evolution of finite-area heton pairs for $a=0.35, \gamma=1.5$ and (a) $b=2.35$, (b) $b=2.4435$ and (c) $b=2.45$ (circle markers in figure $9(\mathrm{a})$ ).

At $a=0$ (figure $14(\mathrm{c})$ ), the vortex structure is really a colinear Z-shaped tripole. The shear on the central vortices is now slightly weaker, so that these vortices elongate but do not break. As explained by Carton and Legras (1994), the elongated central vortex rotates as fast as the two satellite anticyclones, so that the large axis of the central ellipse remains perpendicular to their intercentroid axis. This maintains at all times the shear on the central vortex below the critical value for breaking. This central vortex adjusts to an elliptical shape by shedding filaments. After adjustment, a Z-shaped tripole emerges. 
(a)
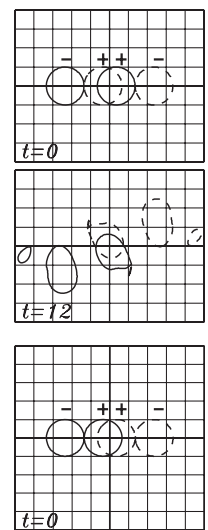

(b)
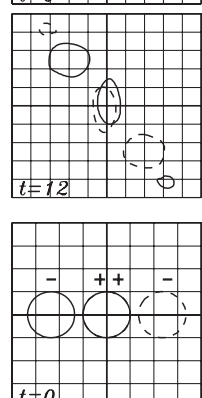

(c)

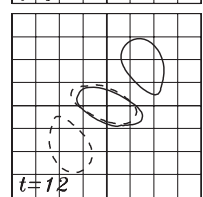

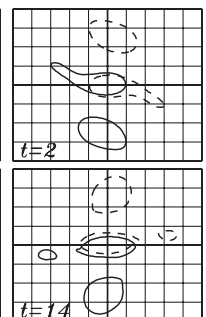
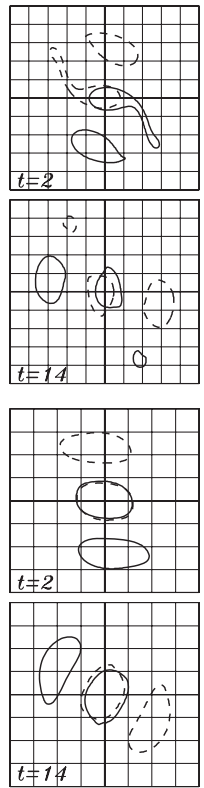
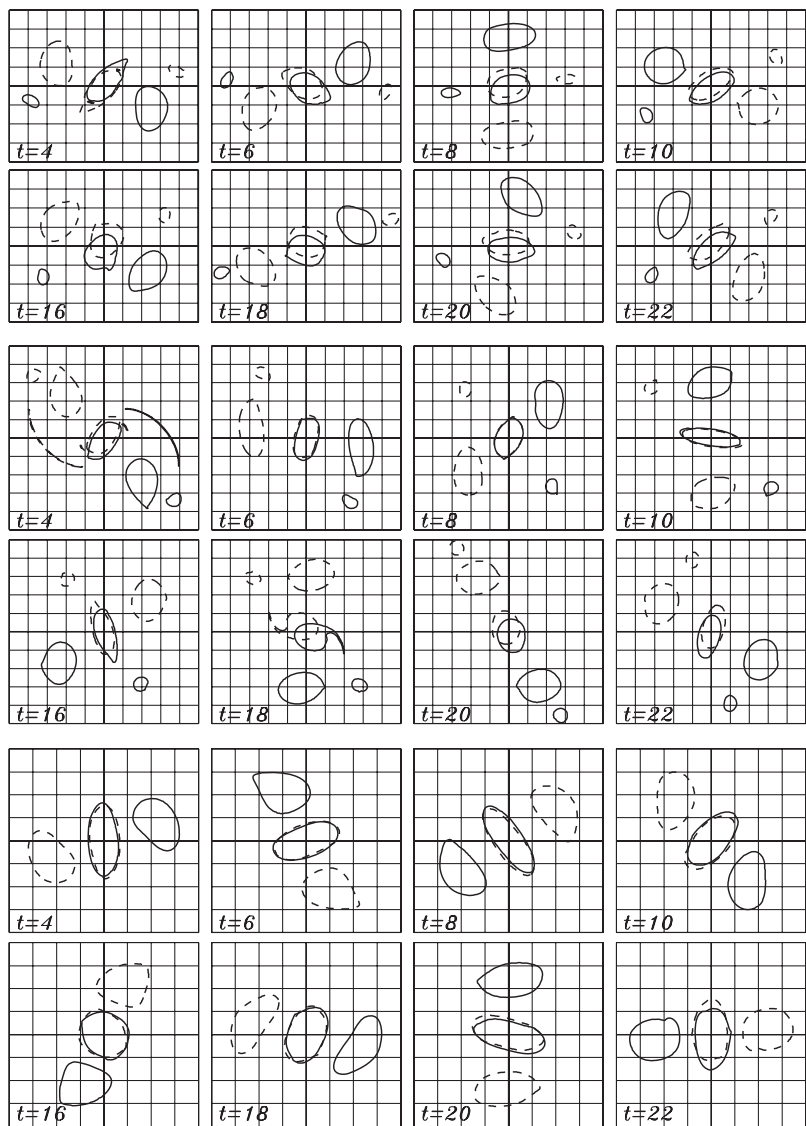

Figure 14. Formation of a Z-shaped tripole for $b=2.35, \gamma=2$ and (a) $a=0.35$, (b) $a=-0.35$ and (c) $a=0$ (box markers in figures 9(a), (c) and (b), respectively.)

The last question that we address here is whether finite-area vortex configurations can remain colinear at all times, even when $a \neq 0$. Obviously, dispersion equation (14), obtained for point vortices, cannot be satisfied here, but such a configuration can be searched numerically.

Figure 15 shows one such case of steady colinear configuration. Figure 16 confirms stationarity by displaying the radius and angle of the central and peripheral vortices. This solution is therefore a Z-shaped tripole with $a \neq 0$.

\subsection{Baroclinic tripole formation from heton collision}

Z-shaped tripoles shown in figures 11(e), 13(c), 14 and 15 were formed from initially colinear configurations of circular vortex patches. The invariance of vortex configurations was checked numerically over long periods (several hundred units of non-dimensional time). As a consequence, such configurations can be regarded as invariant over their typical lifetimes in planetary fluids. The question addressed here is the possible formation of a Z-shaped tripole from the head-on collision of two hetons. Indeed, it is a well-known result (Carton 1988) that two-dimensional tripoles can form from the head-on collision of two dipoles. 

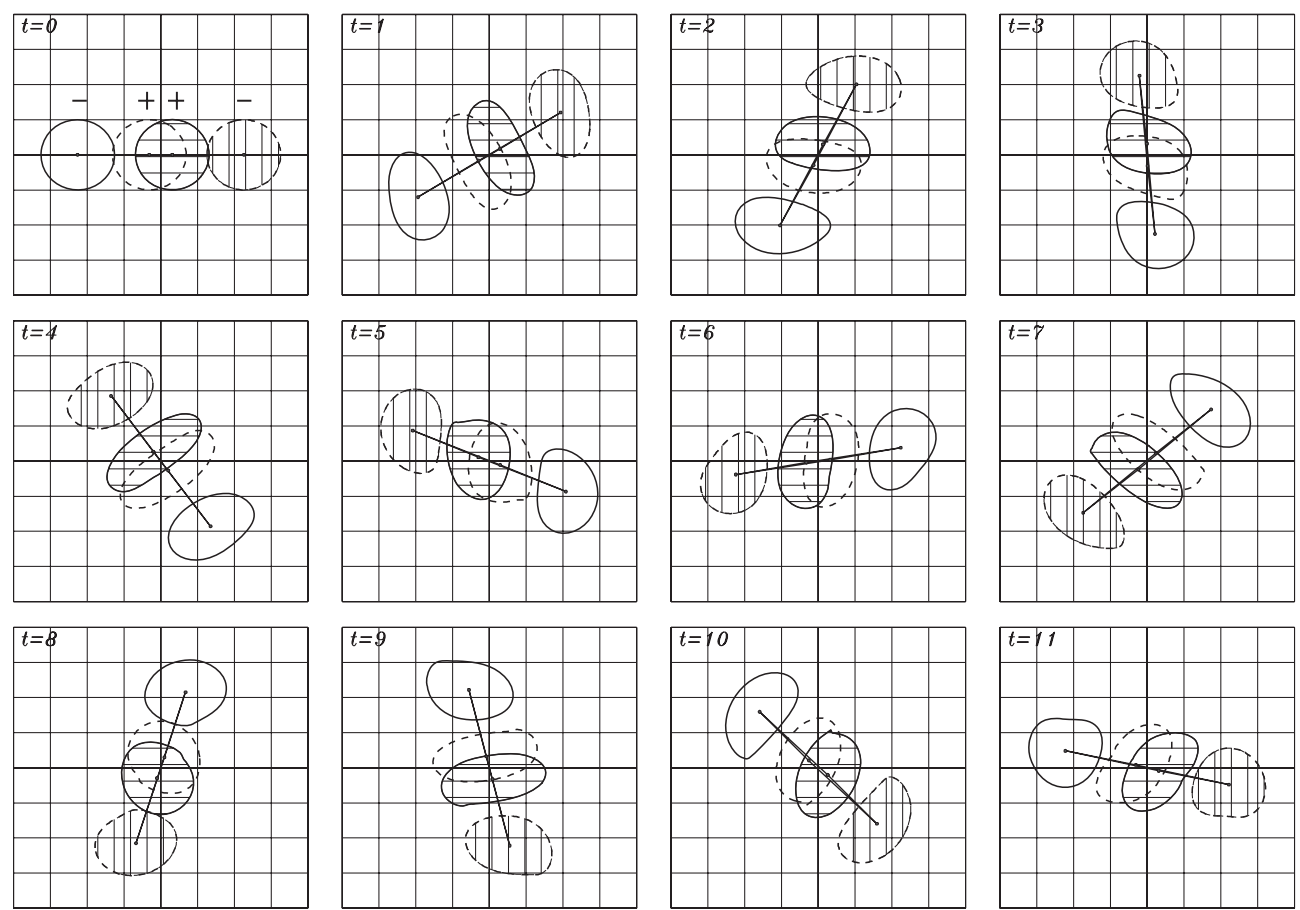

Figure 15. Colinear vortex structure motion at $a=0.311, b=2.2576$ and $\gamma=1$ (an analogue to figure $7(a)$ ). Vortex $\left(\begin{array}{l}1 \\ 1\end{array}\right)$ has horizontal filling and $\left(\begin{array}{l}2 \\ 2\end{array}\right)$ vertical filling.

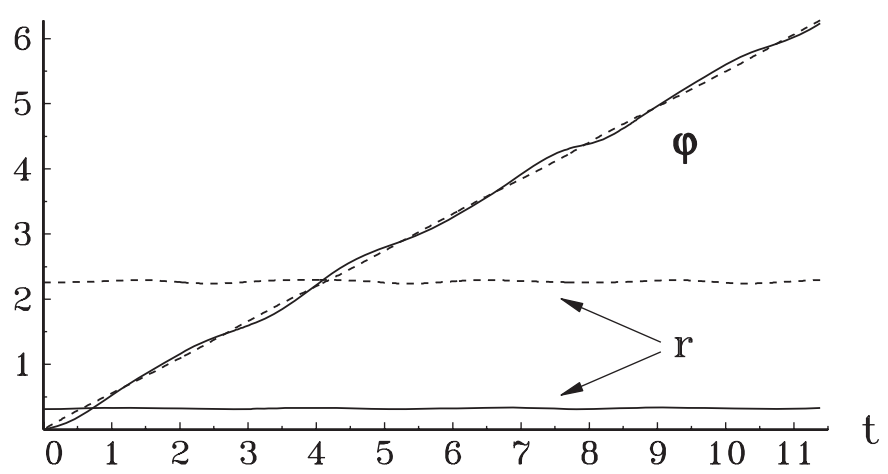

Figure 16. Radius vector, $r(t)$, and angle, $\varphi(t)$, between $r$ and the $x$-axis for the centers of fills' vortices $\left(\begin{array}{l}1 \\ 1\end{array}\right)$ (solid lines) and $\left(\begin{array}{l}2 \\ 2\end{array}\right)$ (dashed lines) in the colinear structure of figure 15 .

Figure 17 presents the regime diagram (in particular, for tripolar structures) for these collisions with initial distance $2 c$ between the two hetons along the $y$-axis; here $c=4$. Unlike previous cases, here $b$ is the distance between the vortex centers in each heton. Initially, for all these calculations, the cyclonic vortices of the upper and lower layers lie along the $y$-axis in the upper and lower semi-planes, respectively, and the anticyclonic vortex patches of the lower and upper layers belong to the left and right semi-planes (see figures 18 and 19). The transition between regimes HD and TD for point vortices dynamics is superimposed on figure 17, indicating a qualitative similarity between point vortex dynamics and finite-area 
$\mathrm{b}$

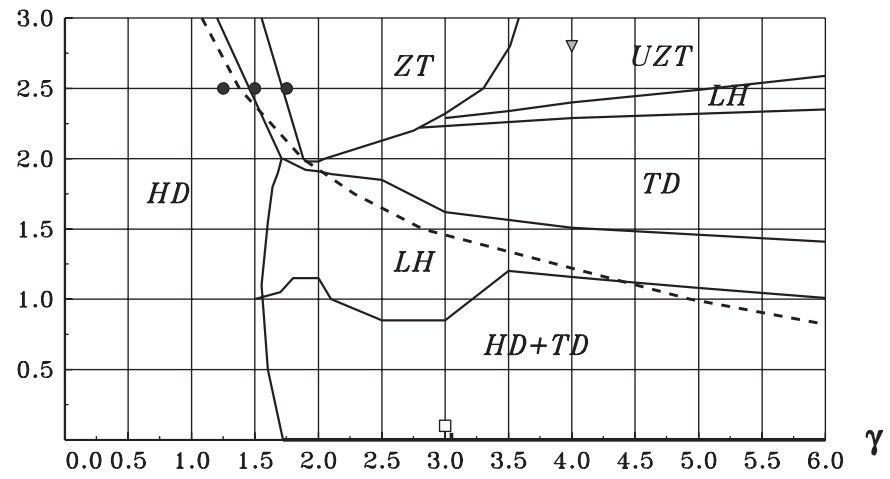

Figure 17. Regime diagram of the finite-area heton pair head-on collision in the $(\gamma, b)$ parameter plane for $a=0$ and $c=4$ : HD is horizontal dipole formation, TD is vertically tilted dipole formation, HD + TD is a combination of horizontal dipole and vertically tilted dipole, ZT is Z-shaped tripole formation, LH is L-shaped heton formation and UZT is an unstable Z-shaped tripolar structure. The dashed line represents the boundary between regions $\{1\}$-analogue of HD and $\{2\}$ - analogue of TD for the point heton pairs (left and right, respectively). This boundary was determined numerically using point vortex theory. The meaning of the markers is explained in the captions of figures 18,19 and 20.

(a)

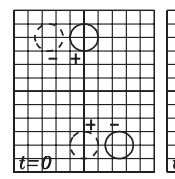

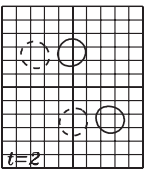
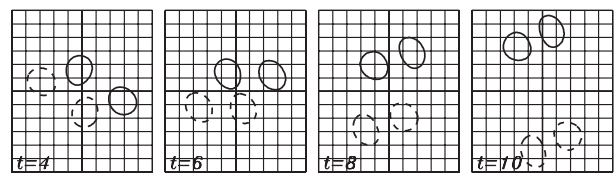

(b)
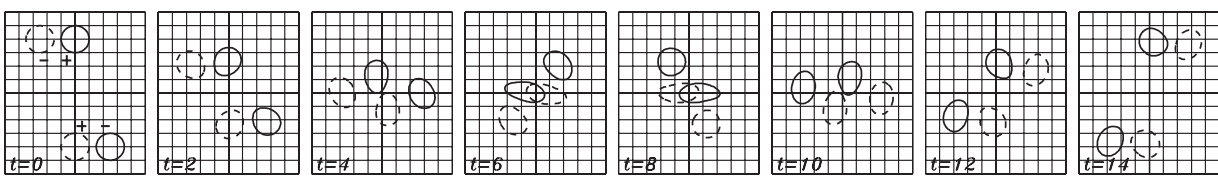

(c)
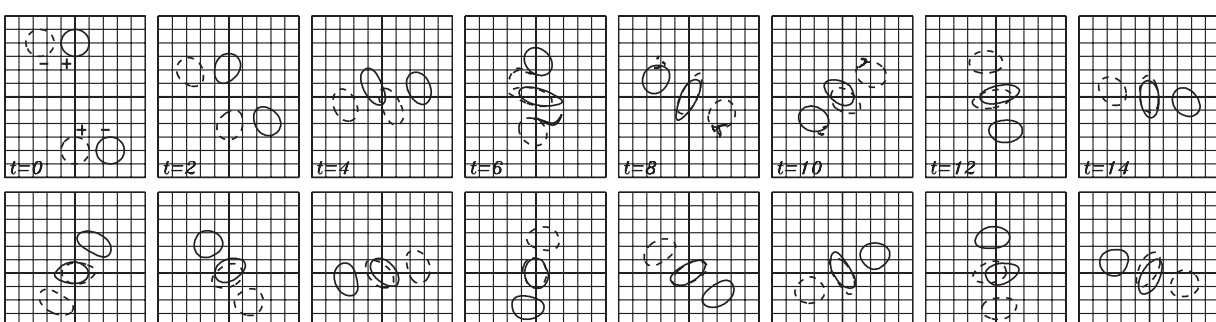

$t=8$ -

Figure 18. Head-on collision of the heton pair at $a=0, b=2.5, c=4$ and (a) $\gamma=1.25$, type HD, (b) $\gamma=1.5$, type TD, and (c) $\gamma=1.75$, type ZT (circle markers in figure 17).

vortex dynamics: predominantly intra-layer interactions occur for low values of $\gamma$ (or of $b$ ), whereas predominantly inter-layer interactions occur for large values of $\gamma$ and $b$.

The main dynamical regimes are illustrated by three examples (figure 18). For moderate layer coupling $(\gamma=1.25)$, the internal vortices are exchanged between the two hetons (figure 18(a)). The intra-layer interaction of vortices is predominant. The final state is a pair of horizontal dipoles along the $y$-axis. For stronger layer coupling $(\gamma=1.5$, figure 18(b)), 

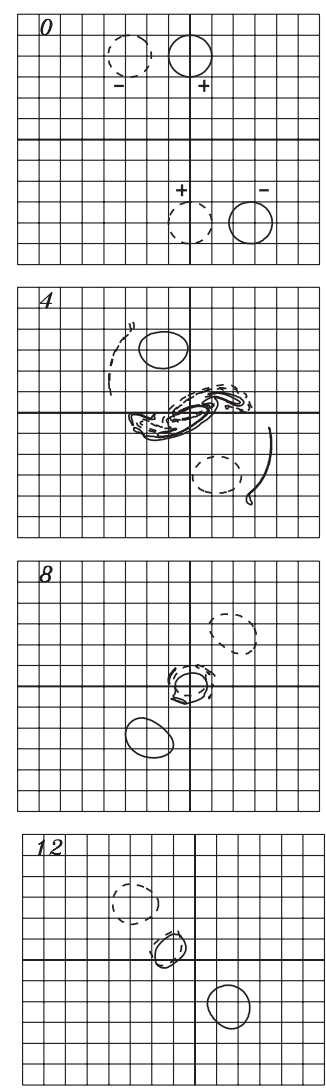
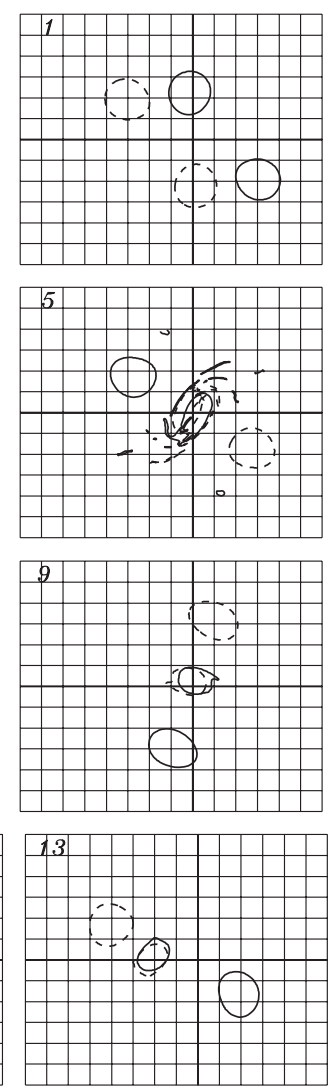
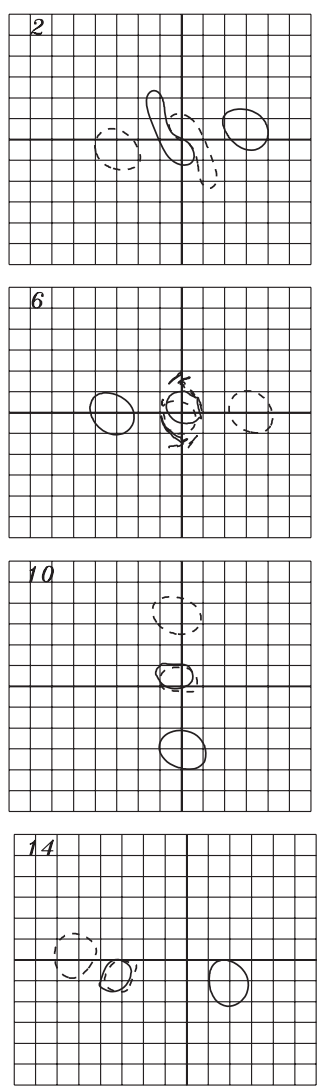
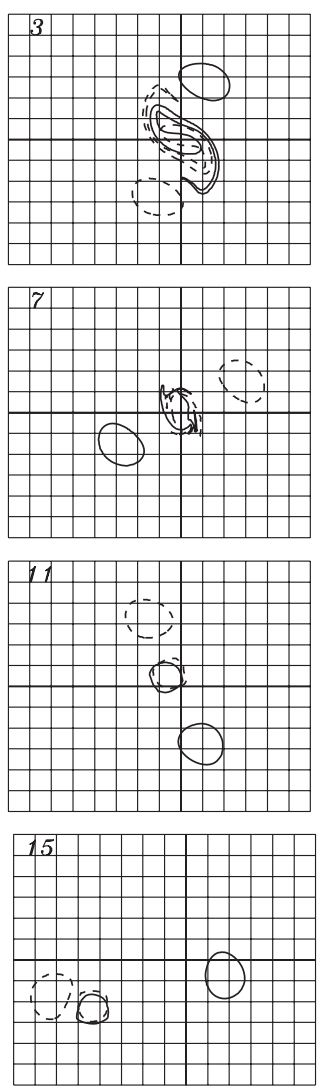

Figure 19. Same as figure 18, but for $\gamma=4$ and $b=2.8$ (triangle marker in figure 17). Type UZT vortex evolution.

exchange of partners does not occur, and vertical dipoles rotate by about $180^{\circ}$ and drift away. Finally, as $\gamma$ is increased again $(\gamma=1.75$, figure 18(c)), a Z-shaped tripole is formed. Thus, $\mathrm{Z}$-shaped tripoles can be formed from a variety of initial configurations of two opposite-signed hetons with zero total impulse. Therefore, it is an important type of baroclinic tripole.

An example of an unstable Z-shaped configuration is given in figure 19 for a case of relatively weak stratification. After the formation of a tripolar structure, the central antiheton is captured by one of the peripheral anticyclones. Then, a transition occurs to a vortex array composed of an L-shaped structure, moving away from the center, and a quasi-static isolated anticyclone. In this case, the dissipation of the central vortices reaches more than $40 \%$ because of strong filamentation. Due to weak stratification, the shear exerted by peripheral vortices on the central vortices prevails on the vertical alignment of the latter; this explains why the tripolar vortex is unstable. Note that the existence of unstable tripolar vortices has already been evidenced by the laboratory experiments of Flór and van Heijst (1996).

Figure 20 displays a vortex evolution of the type HD+TD. During the initial stage, the main process is the instability of hetons with mode $m=3$. They form two small-scale hetons with tilted axis, which move toward each other, and four non-compensated two-layer vortices, which, by pairs, move along arched trajectories. The non-compensated two-layer vortices collide by pairs and, after a merger of vortices in each layer, form two hetons running away along the $y$-axis. The other two hetons, after colliding, transform into two horizontal 

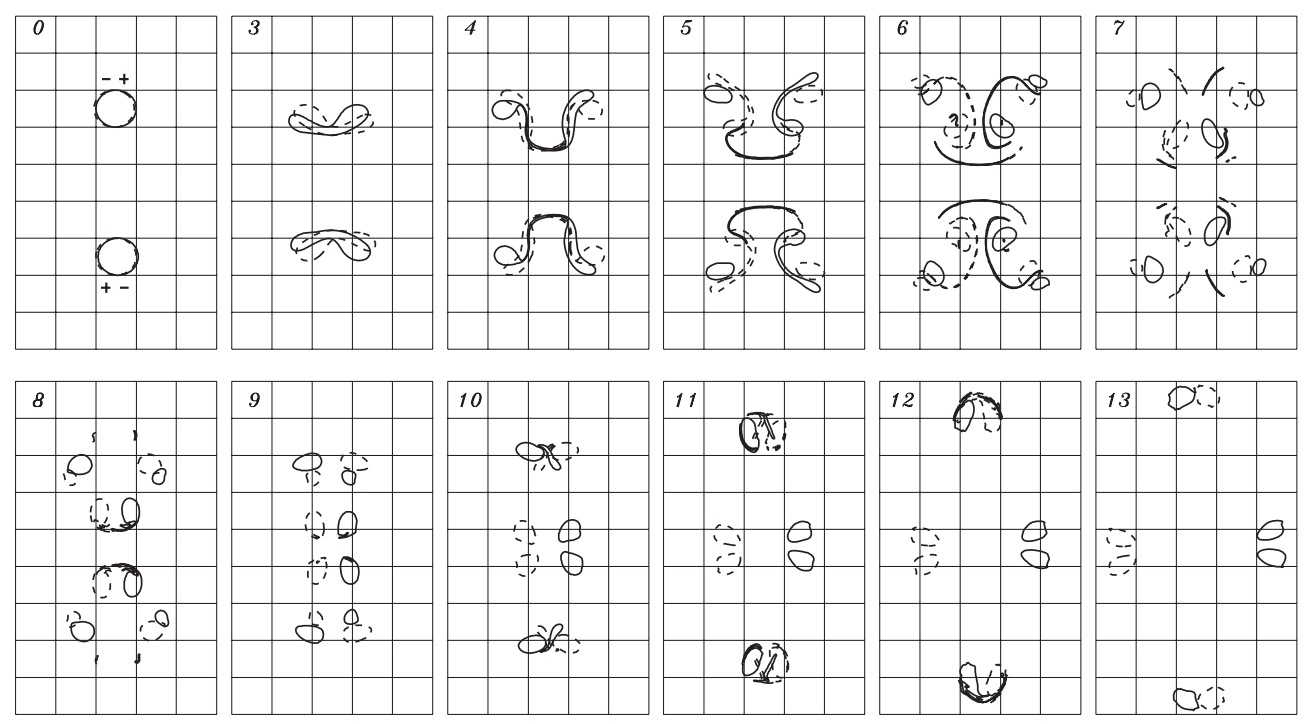

Figure 20. Same as figure 18, but for $\gamma=3$ and $b=0.1$ (box marker in figure 17). Type HD+TD vortex evolution.

dipoles drifting apart. The formation of two horizontal and two vertical dipoles, which scatter practically at the right angle, is the result of such a multistage interaction.

\section{Conclusions}

\subsection{Main results}

The finite-time evolution of a heton pair with zero linear impulse was studied analytically and numerically for various values of angular momentum.

For point vortices, the conservation of five integral quantities ensures the integrability of vortex motion. Specific solutions can be obtained analytically, in particular for weak and strong layer coupling. Then, the possible vortex trajectories were classified using trilinear coordinates.

For weak layer coupling, trajectories are open and correspond to horizontal dipoles. For moderate layer coupling, trajectories are still open but a transition between horizontal dipoles and hetons is possible. For even stronger layer coupling, a regime of closed, periodic, trajectories appears.

In physical space, closed trajectories correspond to central vortices closer to the center of the plane as $\gamma$ increases (if they were initially located at this center). Therefore, a pointwise baroclinic tripole, which is Z-shaped, is possible at large enough $\gamma$. Furthermore, we have shown the existence of a new, stationary, pointwise vortex compound: a four-vortex structure in 'solid-body' rotation ${ }^{3}$ Point vortex trajectories in the rotating frame of reference also evidence four-vortex states with periodic motion of the central vortices.

Finally, as indicated by the trilinear coordinate regime diagram, the transition between tripole, horizontal dipole and hetonic regimes also depends on the initial tilt of the central vortices (parameter $a$ ).

3 Recently, Kizner (2006) obtained a family of translating stationary solutions for four vortices in a two-layer fluid, called 'heton quartets'. 
To reach more realism, the interaction between two finite-area, opposite-signed hetons was studied numerically.

Firstly, as layer coupling is increased, numerical simulations evidence a transition between open trajectories of horizontal dipoles, or mixed heton-horizontal dipoles (called Lshaped dipoles), to closed trajectories of baroclinic (Z-shaped) tripoles, when the two central cyclones were initially aligned. This shows the similarity between point vortex dynamics and finite-area vortex dynamics, as long as vortex splitting or a merger is not involved. The formation of an L-shaped heton is specifically a finite-area evolution, since it results from the breaking of the central cyclones under the shear exerted by the peripheral anticyclones.

Secondly, increasing the separation between the central cyclones diminishes their interaction and favors the formation of horizontal dipoles. The formation of a Z-shaped tripole is therefore favored by small initial distances between the central cyclones and by strong layer coupling. Since they form from initial conditions different from their final state, Z-shaped tripoles appear as attractors for a variety of initial conditions.

Thirdly, oscillating states, intermediate between horizontal dipoles and the Z-shaped tripole, also occur.

In summary, this study has not only classified and explained the nonlinear regimes of heton interaction, initially colinear or distant, but has also evidenced two robust vortex compounds of baroclinic flows: the L-shaped heton and the Z-shaped tripole.

Figures 9 and 17 have shown the similarity between the dynamics of finite-core hetons and of point hetons, although the former are more complex than the latter. This similarity is based on the strength of layer coupling and on the interactions between the satellite and the central vortices. These influences have been detailed for each part of the study.

\subsection{Relation to previous work and perspective}

Referring now to the Corréard and Carton (1998) study, which was one of the motivations for this study, we have rationalized here all the vortex evolutions that were observed then. This rationalization lies both on our point vortex study, which provides the domain of existence of closed and open trajectories, and on the nature of the vortex associations that prevail in each part of the parameter plane: formation of hetons, horizontal dipoles and tripoles. Furthermore, the present study confirms that these dynamical regimes also hold for finite-area heton pairs and that new vortex compounds (the L-shaped dipole) form only from finite-area heton interactions.

The two main differences between our work and that of Correard and Carton are the uniformity of potential vorticity inside the vortex disks and the vertical symmetry of the potential vorticity distribution, which exist here and not in the former study. Nevertheless, both studies conclude that the Z-shaped tripole is a long-lived vortex compound in rotating stratified flows. Therefore, although the Corréard and Carton study was conducted in a less systematic manner than the present study, it then provides evidence that vertically asymmetric and horizontally non-uniform, opposite-signed heton pairs display dynamical regimes similar to those of symmetric and uniform heton pairs.

We also put our present results in perspective with other former studies (Correard and Carton 1999, Sokolovskiy and Verron 2000a, 2000b, 2002a, 2002b). These studies have evidenced how T-, $\Lambda$ - and $\Pi$-shaped tripoles can originate from the instability of baroclinic vortices. Previous studies also showed that $\Lambda$-shaped tripoles (also called 'roundabout') can originate from the head-on collision of like-signed hetons (Sokolovskiy 1989, Sokolovskiy and Verron 2000b). In that interaction, two like-signed vortices merge in the same layer and form a larger core, while opposite-signed vortices become satellites. 'Roundabout' tripoles 
can also form from the instability of a single heton, but only when $h_{1} q_{1}+h_{2} q_{2} \neq 0$ (Kozlov et al1986, Sokolovskiy and Verron 2000a). Here, it was shown that Z-shaped tripoles can originate from the head-on collision of opposite-signed hetons.

The classification of these various forms of tripoles and the determination of their domain of stability should be carried out in a further study, along with that of more complex baroclinic multipoles (quadrupoles, pentapoles, etc). This study should provide a map of possible transitions between baroclinic monopoles, dipoles and multipoles, as was obtained for twodimensional vortices by Morel and Carton (1994).

\subsection{Observations of baroclinic multipoles}

Another justification for our study was the existence and the role of hetons in planetary fluids. It must be noted that baroclinic multipoles, such as those evidenced by the present study, have also been observed in the oceans. A baroclinic tripole was observed in the Bay of Biscay, forming from the instability of the Navidad Current on the Cape Ferret Canyon (Pingree and Le Cann 1992). The association of meddies (Mediterranean water eddies), intensified at mid-depth of the northeastern Atlantic Ocean, with Mediterranean water cyclones of much larger vertical extent is an illustration of L-shaped hetons. Such vortex compounds are relatively stable in the ocean, since their trajectory was followed for 6 months (compared to a 5-day internal rotation period). These examples suggest that the formation and life-cycle of baroclinic multipoles should also be studied in the presence of a mean flow.

\section{Acknowledgments}

XC acknowledges support from UBO and from SHOM (French Navy Oceanographic Service) for this study. MAS was supported by grant nos. 07-05-92210, 07-05-00452 and 08-05-00061 from RFBR (Russian Foundation for Basic Research) and no. 09-01-92504/RUM1-2943-R009 from RFBR/CRDF, and by the Program 'The development of the scientific potential of higher school' No. 2.1.1/554.

The work is a contribution to the INTAS contract no. 7297 'Vortex Dynamics' (collaborative call with Airbus). This investigation was also conducted within the frames of the European Research Group 'Regular and chaotic hydrodynamics'.

The authors acknowledge the fruitful remarks by the anonymous referees, which helped improve this paper.

\section{References}

Aref H and Stremler M A 1999 Four-vortex motion with zero total circulation and impulse Phys. Fluids 11 3704-15 Baey J M and Carton X 2002 Vortex multipoles in two-layer rotating shallow-water flows J. Fluid Mech. 460 151-75 Borisov A V and Mamaev I S 2005 Mathematical Methods of Dynamics of Vortex Structures (Moscow-Izhevsk: Institute of Computer Science)

Carton X 1988 Stabilité des structures cohérentes mono- et multipolaires dans les écoulements 2D et quasigéostrophiques Thèse de doctorat de l'Université $\mathrm{P}$ et M Curie, Paris

Carton X 2001 Hydrodynamical modeling of oceanic vortices Surv. Geophys. 22 179-263

Carton X J and Corréard S M 1998 Baroclinic tripolar vortices: formation and subsequent evolution Simulation and Identification of Organized Structures in Flows (IUTAM/SIMFLOW Symp.) ed J N Sorensen, E J Hopfinger and N Aubry (Dordrecht: Kluwer) pp. 181-90

Carton X J and Legras B 1994 The life-cycle of tripoles in two-dimensional incompressible flows J. Fluid Mech. $26753-82$ 
Carton X J and McWilliams J C 1989 Barotropic and baroclinic instabilities of axisymmetric vortices in a QG model Mesoscale/Synoptic Coherent Structures in Geophysical Turbulence (Elsevier Oceanographic Series vol 50) ed J C J Nihoul and B M Jamart (Amsterdam: Elsevier) pp. 225-4

Carton X J and McWilliams J C 1996 Nonlinear oscillatory evolution of a baroclinically unstable geostrophic vortex Dyn. Atmos. Oceans 24 207-14

Chao S-Y and Shaw P-T 1999a Close interactions between two pairs of heton-like vortices under sea ice J. Geophys. Res. 104 23591-605

Chao S-Y and Shaw P-T 1999b Fission of heton-lake vortices under sea ice J. Oceanogr. 55 65-78

Chao S-Y and Shaw P-T 2000 Slope-enhanced fission of salty hetons under sea ice J. Phys. Oceanogr. 30 2866-82

Chao S-Y and Shaw P-T 2003 Heton shedding from submarine-canyon plumes in an Arctic boundary current system: sensitivity to the undercurrent J. Phys. Oceanogr. 33 2032-44

Chérubin L et al 1997 Descriptive analysis of the hydrology and currents on the Iberian shelf from Gibraltar to cape Finisterre: preliminary results of the INTERAFOS and SEMANE experiments Ann. Hydrogr. 21 5-69

Chérubin L, Carton X and Dritschel D G 2007 Vortex dipole formation by baroclinic instability of boundary currents J. Phys. Oceanogr. 37 1661-77

Corréard S M and Carton X J 1998 Vertical alignment of geostrophic vortices: on the influence of the initial distribution of potential vorticity Simulation and Identification of Organized Structures in Flows (IUTAM/SIMFLOW Symp.) ed J Sorensen, E Hopfinger and N Aubry (Dordrecht: Kluwer) pp. 191-200

Corréard S M and Carton X J 1999 Formation and stability of tripolar vortices in stratified geostrophic flows. Nuovo Cimento C 22 767-77

Flatau M, Schubert W H and Stovens D E 1994 The role of baroclinic processes in tropical cyclone motion: the influence of vertical tilt J. Atmos. Sci. 51 2589-601

Flierl G R 1988 On the instability of geostrophic vortices J. Fluid Mech. 197 349-88

Flierl G R, Carton X and Messager C 1999 Vortex formation by unstable oceanic jets Eur. Ser. Appl. Ind. Math. 7 137-50

Flór J B and van Heijst G J F 1996 Stable and unstable monopolar vortices in a stratified fluid J. Fluid Mech. $311257-87$

Gryanik V M 1983a Dynamics of singular geostrophic vortices in a two-layer model of the atmosphere (ocean) Izv. Atmos. Ocean. Phys. $19227-40$

Gryanik V M 1983b Dynamics of localized vortical disturbances-'vortex charges' in a baroclinic fluid Izv. Atmos. Ocean. Phys. 19 467-75

Gryanik V M, Sokolovskiy M A and Verron J 2006 Dynamics of heton-like vortices Reg. Chaot. Dyn. 11 $383-434$

Hairer E, Nørsett S R and Wanner G 1987 Solving Ordinary Differential Equations (Berlin: Springer)

Helfrich K R and Send U 1988 Finite-amplitude evolution of two-layer geostrophic vortices J. Fluid Mech. 197 $331-48$

Hogg N G and Stommel H M 1985a The heton, an elementary interaction between discrete baroclinic geostrophic vortices, and its implication concerning eddy heat-flow Proc. R. Soc. A 397 1-20

Hogg N G and Stommel H M 1985b Hetonic explosions: the breakup and spread of warm pools as explained by baroclinic point vortices J. Atmos. Sci. 42 1465-76

Jamaloodeen M I and Newton P K 2007 Two-layer quasigeostrophic potential vorticity model J. Math. Phys. 48 1-48

Kizner Z 2006 Stability and transitions of hetonic quartets and baroclinic modons Phys. Fluids 18056601

Kozlov V F 1983 The method of contour dynamics in model problems of the ocean topographic cyclogenesis $I z v$. Atmos. Ocean. Phys. $19635-40$

Kozlov V F 1994 Geophysical hydrodynamics of vortex patches Mar. Hydrophys. J. 1 26-35

Kozlov V F and Makarov V G 1985 Simulation of the instability of axisymmetric vortices using the contour dynamics method Fluid Dyn. 20 28-34

Kozlov V F, Makarov V G and Sokolovskiy M A 1986 A numerical model of baroclinic instability of axially symmetric vortices in a two-layer ocean Izv. Atmos. Ocean. Phys. 22 868-74

Legg S and Marshall J 1993 A heton model of the spreading phase of open-ocean deep convection J. Phys. Oceanogr. 23 1040-56

Makarov V G 1991 Computational algorithm of the contour dynamics method with changeable topology of domains under study Model. Mech. 5 83-95

McWilliams J C 1984 The emergence of isolated coherent vortices in turbulent flows J. Fluid Mech. 146 21-43

McWilliams J C 1989 Statistical properties of decaying geostrophic turbulence J. Fluid Mech. 198 199-230

McWilliams J C 1990a The vortices of two-dimensional turbulence J. Fluid Mech. 219 361-85

McWilliams J C 1990b The vortices of geostrophic turbulence J. Fluid Mech. 219 387-404

Meacham S P 1991 Meander evolution on piecewise-uniform, quasi-geostrophic jets J. Phys. Oceanogr. 21 1139-70

Morel Y G and Carton X J 1994 Multipolar vortices in two-dimensional incompressible flows J. Fluid Mech. 267 23-51 
Morel Y and McWilliams J C 2001 Effect of isopycnal and diapycnal mixing on the stability of oceanic currents J. Phys. Oceanogr. 31 2280-96

Orlandi P and van Heijst G J F 1992 Numerical simulations of tripolar vortices in 2D flows Fluid Dyn. Res. 9179-206

Paillet J, Le Cann B, Carton X, Morel Y and Serpette A 2002 Dynamics and evolution of a northern meddy J. Phys. Oceanogr. 32 55-79

Pedlosky J 1985 The instability of continuous heton clouds J. Atmos. Sci. 42 1477-86

Pedlosky J 1987 Geophysical Fluid Dynamics 2nd edn (Berlin: Springer)

Pingree R and Le Cann B 1992 Three anticyclonic Slope Water Oceanic eDDIES (SWODDIES) in the southern Bay of Biscay in 1990 Deep-Sea Res. 39 1147-75

Polvani L M and Carton X J 1990 The tripole: a new coherent vortex structure of incompressible 2D flows Geophys. Astrophys. Fluid Dyn. 51 87-102

Polvani L M 1991 Two-layer geostrophic dynamics. Part 2. Vertical alignment and two-layer V-States J. Fluid Mech. 225 241-70

Pullin D I 1992 Contour dynamics method Annu. Rev. Fluid Mech. 24 89-115

Reznik G and Kizner Z 2007 Two-layer quasi-geostrophic singular vortices embedded in a regular flow. Part 1. Invariants of motion and stability of vortex pairs J. Fluid Mech. 584 185-202

Sokolovskiy M A 1989 Head-on collisions of distributed hetons Trans. Dokl. USSR Acad. Sci. 306 215-7

Sokolovskiy M A 1997a Stability of an axisymmetric three-layer vortex Izv. Atmos. Ocean. Phys. 33 16-26

Sokolovskiy M A 1997b Stability analysis of the axisymmetric three-layered vortex using contour dynamics method. Comput. Fluid Dyn. J. 6 133-56

Sokolovskiy M A and Verron J 2000a Finite-core hetons: stability and interactions J. Fluid Mech. 423 127-54

Sokolovskiy M A and Verron J 2000b Four-vortex motion in the two-layer approximation: Integrable case Reg. Chaot. Dyn. 5 413-36

Sokolovskiy M A and Verron J 2002a New stationary solutions of the three-vortex problem in a two-layer fluid Dokl. Phys. 47 233-7

Sokolovskiy M A and Verron J 2002b Dynamics of the triangular two-layer vortex structures with zero total intensity Reg. Chaot. Dyn. 7 435-72

Sokolovskiy M A and Verron J 2004 Dynamics of three vortices in a two-layer fluid Reg. Chaot. Dyn. 9 417-28

Sokolovskiy M A and Verron J 2006 Some properties of motion of $A+1$ vortices in a two-layer rotating fluid. Russ. Sci. J. Nonlinear Dyn. 2 27-54

Sokolovskiy M A, Verron J and Vagina I M 2001 Effect of a submerged small-height obstacle on the dynamics of a distributed heton Izv. Atmos. Ocean. Phys. 37 131-43

Takahashi J and Masuda A 1998 Mechanism of southward translation of meddies J. Oceanogr. 54 669-80

Tevs M V 1999 A kinematic study of the vertical structure of tropic cyclones based on an $N$-level quasi-geostrophic atmospheric model Izv. Atmos. Ocean. Phys. 35 481-6

Valcke S and Verron J 1993 On interactions between two finite-core hetons Phys. Fluids A 5 2058-60

Verron J, Hopfinger E J and McWilliams J C 1990 Sensitivity to initial conditions in the merging of two-layer baroclinic vortices Phys. Fluids A 2 886-9

Verron J and Valcke S 1994 Scale-dependent merging of baroclinic vortices J. Fluid Mech. 264 81-106 